\title{
Bioinspired Mobility-Aware Clustering Optimization in Flying Ad Hoc Sensor Network for Internet of Things: BIMAC-FASNET
}

\author{
Abdu Salam (D), ${ }^{1}$ Qaisar Javaid, ${ }^{1}$ and Masood Ahmad ${ }^{2}$ \\ ${ }^{1}$ Department of Computer Science \& Software Engineering, International Islamic University, Islamabad, Pakistan \\ ${ }^{2}$ Department of Computer Science, Abdul Wali Khan University, Mardan, Pakistan \\ Correspondence should be addressed to Abdu Salam; abdusalam.phdcs148@iiu.edu.pk
}

Received 15 July 2020; Revised 8 August 2020; Accepted 24 August 2020; Published 7 September 2020

Academic Editor: Atif Khan

Copyright (c) 2020 Abdu Salam et al. This is an open access article distributed under the Creative Commons Attribution License, which permits unrestricted use, distribution, and reproduction in any medium, provided the original work is properly cited.

Flying ad hoc sensor network (FASNET) for Internet of Things (IoT) consists of multiple unmanned aerial vehicles (multi-UAVs) with high mobility, quick changes in topology, and diverse direction. The flying multi-UAVs were operated remotely by human beings or automatically by an onboard system. The applications of multi-UAVs are remote sensing, tracking, observing, and monitoring. It has a different nature compared to ordinary ad hoc network. The speed and diverse directions of multi-UAVs make it harder to route information in a desired way. Different issues may arise due to differences in unmanned aerial vehicle mobility, speed, diverse direction, and quick changes in topology. The researchers proposed conventional ad hoc routing protocols which has poor aspects for the flying ad hoc networks. They tried to tackle the issue by using the clustering approach that divides the network structure into multiple clusters, each with its own cluster head $(\mathrm{CH})$. During the selection of $\mathrm{CH}$ and balance cluster formation, they consider only location awareness, neighborhood range, residual energy, and connection to the base station (BS) while ignoring the multi-UAVs distance, speed, direction, degree, and communication load. In this paper, we proposed bioinspired mobility-aware clustering optimization scheme based on bee intelligence foraging behavior for routing, considering relative mobility, residual energy, degree, and communication load during $\mathrm{CH}$ selection and balanced cluster formation. First, the clustering problem in network is formulated to dynamic optimization problem. An algorithm is designed based on bee intelligence, applied to select optimal UAVs $\mathrm{CH}$ and balanced cluster. The simulation results show that the proposed BIMACFASNET scheme performs better among existing clustering protocols in terms of link-connection lifetime, reaffiliation rate, communication load, number of UAVs per cluster, $\mathrm{CH}$ lifetime, and cluster formation time.

\section{Introduction}

Flying ad hoc sensor network (FASNET) for Internet of Things (IoT) consists of multiple flying nodes, i.e., unmanned aerial vehicles (UAVs) and ground segments (GSs) [1-3]. The flying nodes/sensors may play the role of a router or a sensor or both in a network. The characteristics of the sensor fly are different from the sensors in conventional wireless sensor network (WSN). The high mobility, communication range, and memory of the nodes are limited. Many issues like architecture and routing need to be addressed for the successful implantation of FASNET. The conventional MANET, VANET, and WSN routing protocols may not be applied directly to flying ad hoc sensor networks [4].
In early stages of flying ad hoc sensor networks, a single UAV was used to monitor, control, observe, and sense the objects or environment, but due to failure of a single UAV, no other UAV was available to maintain communication. Today with the use of multi-UAVs, the failure of single UAV does not disturb the communication because multiple UAVs are used to reconfigure and maintain communication among UAVs and GS. Due to the dynamic nature of flying ad hoc sensor network, the UAVs may have the issue of mobility, energy, etc. The advantages of multi-UAV network are that it works in coordination and collaboration, which does not affect the overall performance of network. In multiUAV system, the base station or controller or server receives information from root UAVs. The root UAVs have more 
power and feature to obtain information from the member UAVs in the range.

The exchange of information among multi-UAVs is based on the cluster approach that divides the network structure into multiple clusters, each with its own head, called cluster head $(\mathrm{CH})$. The cluster member nodes send their information to the $\mathrm{CH}$ in an aggregate manner with proper synchronization or organizational structure. Clusterbased routing avoids the collision during communication of nodes. Multihop networks with $\mathrm{CH}$ provide low latency as compared to flat routing. The topology changes in this scheme are adjusted locally within the cluster and do not influence the whole network. In this way, the network becomes more scalable; the topology information aggregates, achieves the routing efficiency, and forms stable and balance network [5].

Cluster-based routing is suitable to provide reliable communication between UAVs and GS. The main objectives are to dynamically update network topology, resolve the issues such as updating routing tables, and deal with highspeed UAVs, which means high mobility.

1.1. Advantages. Cluster-based routing in FASNET is very constructive with following advantages [5]:

(i) Use aggregation approach to obtain information from the multi-UAVs in the cluster by $\mathrm{CH}$ instead of from the whole flying ad hoc network.

(ii) Due to small size of clusters, the intercluster or intracluster movement of nodes updates their routing information locally about the change in topology that show stability and efficiency at node and cluster level.

(iii) Use of clusters reduces the routing overhead at $\mathrm{CH}$ level as compared to flat network structure in which all the nodes communicated through router.

(iv) UAVs under the cluster heads, only communication with their $\mathrm{CH}$ or sometimes with their gateway $\mathrm{CH}$, and the communication of flying UAVs are constrained to preserve communication bandwidth.

(v) Reuse of frequency in the clustering approach that provides communication without collision and nonoverlapping clusters. The unused bandwidth can be utilized for some other beneficial purposes.

1.2. Multi-UAVs Applications in FASNET for IoT. The applications of this emerging area are unlimited. In early stages, the technology that was used to destroy in battlefield is now applied for human being betterment in the variety of fields to minimize the trespass of human. The rising interests of users in the multi-UAVs technology have developed new era of applications.

1.2.1. Reconnaissance and Patrolling. The application of multi-UAVs is to observe the particular area safe and crime free. The UAVs with thermal of hyperspectral sensors capture and monitor the area of interest, objects, and events during patrolling. These UAVs periodically patrol in the given area to observe, inspect, or secure from doubtful or unfamiliar activity. It can detect any type of change in the given range such as weapons, crime, and drugs [6]. During the mission flying nodes in reconnaissance and patrolling, the routes are not predictable because they change randomly their positions and paths according to the situation [7]. The flying nodes cooperate and communicate information with one another, and according to the information received, they change the topology or routing mechanism.

1.2.2. Search and Rescue Operation. The UAV technology is used to search or rescue the lost personnel and hostages in the area that are not easily accessible for human being. The flying UAVs are integrated with thermal or spectral sensors to find the position of lost person [8]. The issue is this area is the UAVs mobility, which is totally based on the type of flying UAV infrastructure. In start of the mission, the predetermined map is used to find the target easily. The flying UAVs are always connected to the BS for sending information in aggregate manner. Real-time communication is needed by flying UAVs in rescue or emergency scenarios that guarantee for information to be sent and received because many obstacles may occur during routing information [9].

1.2.3. Shipping and Delivery. The UAVs have capability to carry payloads. Many of the companies use UAV technology in electronic commerce for shipping and delivering products to improve the quality of their services and decreasing the costs. People get instant services for the ordered products and pizza at homes [8].

1.2.4. Disaster Management. The UAV technology in disaster relief management obtains the required information quickly to predict the incidence of disaster. The human may face difficulties or obstacles that prevent analysis of the entire affected area [8]. The team may be not aware about the condition of ground that may cause the wastage of precious lives and time. Awareness about the environment is based on the multi-UAV technology to observe the issues and make reliable transmission of information to the BS. During the assessment of information, the main aim is to integrate information received from different sources to make a bridge of various information technologies used in disaster management [10].

1.2.5. Engineering and Construction. The multi-UAV technology is used in most of the engineering firms to manage and monitor the projects like installation of transmission line, industry, and airport planning infrastructure and oil pipelines and maintain inspection activities. The unmanned aerial vehicles are used to analyze construction and verify their progress and quality. It is also used to evaluate the conditions of the environment in order to prevent possible calamities [10]. 
1.2.6. Traffic and Urban Monitoring. The application of this emerging area is to monitor or resolve the issue of traffic jam and numerous accidents in complex infrastructure of urban and metropolitan areas [11]. The UAVs easily detect and take information about the road jam, railway track incidents, or street junction with thermal or hyperspectral sensors by capturing real-time situations. The flying UAVs are dependent on one another and send information to the base station because the distance may vary between object/target and base station [12].

FASNET is an emerging area that builds an interconnection among flying sensors (UAVs) and GS. Routing of information in flying node is challenging task and hot issue that took the attention of the research community in the recent years. Although a number of researchers contributed in the reliable communication [13-17], still this area is in its infancy and there are a number of issues existing due to high mobility of flying multi-UAVs.

The rest of the paper is organized as that how to develop mobility-aware cluster-based routing, which are suitable for maximum scenarios and conditions of FASNET. In Section 2 , first we present the review of the existing swarm intelligence optimization algorithms, which exhibit the intelligent behavior of social creatures. Second, the mobility model used in flying ad hoc sensor networks and, third, the clustering protocols based on swarm intelligence are extensively reviewed and also explained to provide optimum cluster organization discussed in detail. The simulation tools and mobility model are analyzed. Section 3 first formulates the clustering problem to dynamic optimization problem. Secondly, the clustering algorithm based on honey bee foraging behavior is presented. Section 4 explains how the mobility-aware $\mathrm{CH}$ is selected and how the balanced clusters are formed. The performance evaluation of reviewed protocols is compared with the proposed scheme in terms of link connection lifetime, UAV speed, communication range, $\mathrm{CH}$ lifetime, UAV degree, reassociation time, and cluster formation time. In the last section, we added the proposed protocol conclusion.

\section{Literature Review}

In this section, the categories of swarm intelligence-based optimization schemes identified are used for mobility-aware clustering in flying ad hoc sensor network. The existing clustering protocols are analyzed with different perspectives, and also the mobility model used for multi-UAVs in flying ad hoc sensor networks is discussed in this section.

\subsection{Swarm Intelligence-Based Optimization (SIO) Schemes.} The exploration and investigation of multiple UAV clustering scheme is an emerging area for future advancement of UAVs technology. The use of multi-UAVs matches with the concept of swarm, which comes from the nature such as the coordination of ants, bees, particles, firefly, wolfs, and frogs. Swarm intelligence means that the intelligent behavior is exhibited by social creatures [18]. The Department of Defense US project ARPA proposed the UAV swarm in 2000 with the program name UAV-SAWR (UAVs Swarm Aerial Warfare Research). The 5S trend, i.e., smart, small, speed, safe, and swarm theory, is presented at CCF-GAIR summit in August 2016 [18]. In this 5S theory, the swarm clustering technique attracted many researchers from both defense and academia. The swarm of UAV activities was performed efficiently by using swarm intelligence optimization algorithm. There are different optimization algorithms based on swarm intelligence including Physarum polycephalum optimization [19], glowworm swarm optimization (GSO) [20], grey wolf optimization (GWO) [21], ant colony optimization (ACO) [22], and bee colony optimization (BCO) [23, 24], as shown in Figure 1.

\subsubsection{Physarum polycephalum Optimization (PPO).} Physarum-inspired network algorithm (PAs) was first time described by Howard in 1931 [25]. Physarum polycephalum is a species of Physarales also called a true slime mold. It has a small single cell and increases the size up to ten centimeters. The plasmodium of PP comprises sponge and tube [19]. The sponge and tube form a network to transform physical and chemical signals information throughout the organism. Change in the topology occurs during exploration of the neighbor environment. The intelligent foraging behavior of Physarum exhibited to solve the different optimization problems in network such as finding the shortest path and minimum risk problem. The authors suggest providing Physarum-inspired network optimization problem with more theoretical bases and improvement in the performance.

2.1.2. Glowworm Swarm Optimization (GSO). The GSO is an optimization algorithm based on swarm intelligence first time introduced by Krishnan and Ghose [20], inspired by the flashing behavior of glowworms. The algorithm is inspired by the functions of natural glowworms. The glowworm works in a group. Each glowworm has luminescence also called luciferin. GSO has five steps: updating luciferin value, selecting neighbor, movement, and updating decision radius [26]. The selection of neighbor is based on the amount or value of luciferin and glowworm location. The value higher than the threshold will be more close to the best solution. GSO can be used to solve the multiconstrained (QoS) multicast routing problem. The issue with GSO compromises on the convergence of speed and accuracy of the fixed step-size.

2.1.3. Grey Wolf Optimization (GWO). GWO inspired by the grey wolf is metaheuristic introduced by Mirjalili et al. [21]. Grey wolves live in groups having a very strict hierarchy, also called packs. Each group has 5-12 members. The hierarchy comprises four levels: alpha $(\alpha)$, beta $(\beta)$, delta $(\delta)$, and omega $(\delta)$. Alpha takes hunting decision based on three principles: first searching, second encircling, and third attacking prey. Beta is a subgroup that helps alpha make hunting decisions, about hunting time, sleeping place, or wake up time. Delta is the subordinate of alpha and beta and 


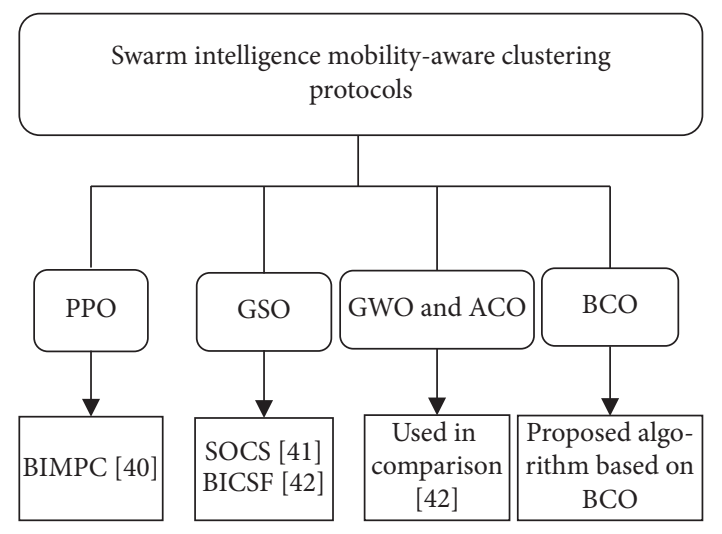

FIgURE 1: Taxonomy of mobility-aware clustering protocols based on swarm intelligence optimization.

submits information about any danger on the boundaries. Omega level is the scapegoat in the pack and submits to all other dominant wolves. Grey wolf optimizer is used to find the optimum solution for problems. The shortcomings of GWO are slow to process accurate problem solving, not good in local search and slow convergence.

2.1.4. Ant Colony Optimization (ACO). The optimization technique ACO is used to discover the shortest path of target by evaluating the ant colony foraging behavior. ACO for the first time was proposed in 1991 by Colorni et al. [22]. It is very effective in solving the problems related to optimization such as traveling salesman problem (TSP), multi-UAVs routing problem, sequential ordering, and other similar optimization problems. ACO has some shortcoming such as complicated theoretical analysis and indeterminate time to convergence.

2.1.5. Bee Colony Optimization (BCO). The optimization technique, bee colony algorithm, is used to find quickly the best food source by swinging or dancing (waggle dance) in the range of food source. During the waggle dance of bees, it makes a colony and exchanges information about the food source. The most common algorithm used for optimization is honey bee mating optimization (HBMO) based on the bee breeding behavior [23]. Another famous optimization algorithm is artificial bee colony optimization $(\mathrm{ABCO})$ proposed in 2005 by Karaboga and others [24]. This algorithm is used to find high-quality food source with optimal solution, by exchanging information and cooperation among the colony bees. For the feasible solution representation, each bee (onlooker, scout, and employed) leaves the colony to search and find the food source. In this paper, we use honeybee foraging behavior to prolong the network lifetime.

2.2. Multi-UAVs Mobility Models. The mobility of UAVs in FASNET is very high, and its model is based on UAVs location, speed, direction, and velocity. The mobility models are planned to provide the complete pattern of mobile UAVs and how these UAVs location, speed, direction, and velocity change over specific time interval. It is used for simulation purposes during evaluation of new protocol. It plays an important role in the performance of multi-UAVs mobilityaware clustering protocols. The mobility models are necessary to match the movement pattern of real-life applications in practical environment. Otherwise, the simulation result and conclusion drawn from analysis for the proposed scheme may be not effective. Some of the mobility models for FASNETs are discussed in this section, each with its own pattern of mobility that will affect the performance of the mobility-aware clustering protocols [27].

We classify the existing mobility models for UAVs protocol simulation in five categories, as shown in Figure 2. The summary of these mobility models is given in Table 1 .

2.2.1. Pure Randomized Mobility Models. The most commonly used category by the research community for network simulation is the pure randomized mobility models in which the multiple UAVs movement are independent and random. The applications of this category are an environmental sensing, traffic, and urban monitoring. The pure randomized mobility models are as follows:

(a) Random waypoint

The random waypoint (RWP) mobility model was first time proposed in 1996 by Johnson and Maltz [28]. In this model, a random movement of mobile UAVs was added with pause time between the UAVs direction and/or speed. The pause time is for few seconds and the UAV then selects the random destination, reaching that destination and then pauses for few seconds and again with selected speed for a random destination in the simulation area. The UAVs freely move in any direction without restriction within a specific area. It means that the UAVs are able to select their destination, direction, and speed independently from adjacent UAV. This model is not suitable for aircraft due to the rapid change in speed and destination. It is not based on the history of the previous movement and neighbor UAVs [29].

(b) Random walk

Random walk (RW) mobility model was first time introduced in 1905 by Pearson [30]. It is proposed to match or emulate the unexpected movement pattern of UAVs in wireless ad hoc networks simulation in [27]. This model is just like RWP mobility model, but the UAV direction and speed change on reaching their target and then, for new time interval, use new direction and speed and so on. This model is memory less and independent from the previous movement and neighbor behavior of UAVs. In some environments, it fails like during mission, the mobile UAVs movement pattern may be constrained due to CHs and its member UAVs [29].

(c) Random direction

The mobility model, random direction (RD), was introduced to resolve the issue in the random 


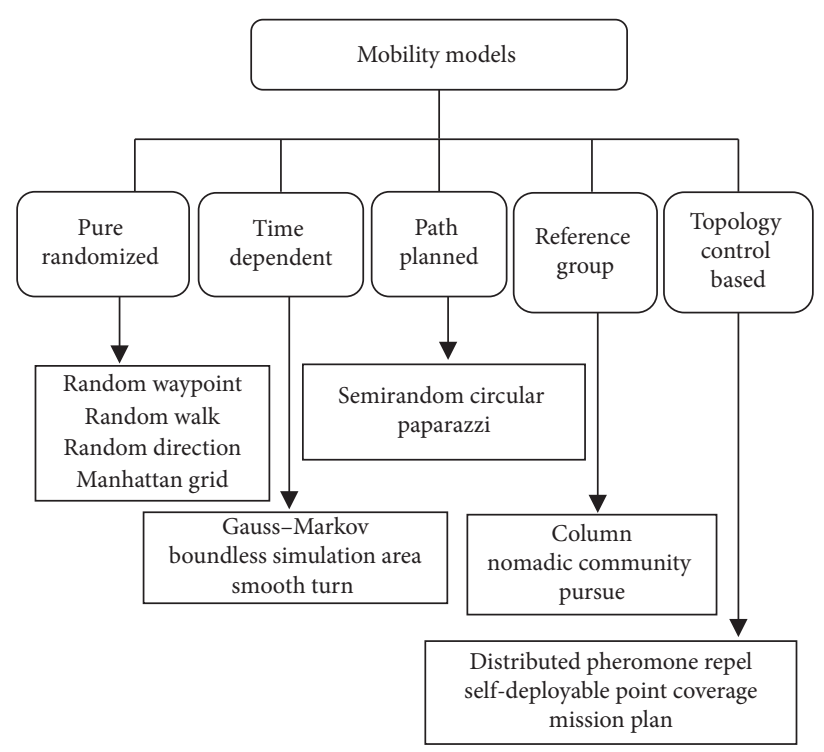

Figure 2: Mobility models for flying ad hoc sensor network.

TABLE 1: Summary of the mobility models and their network characteristics [27].

\begin{tabular}{|c|c|c|c|c|c|c|}
\hline Mobility model & Category & Smooth curve & Smooth acceleration & Microvariation & Connection awareness & Collison avoiding \\
\hline RWP [28] & Random & $x$ & $x$ & $x$ & $x$ & $x$ \\
\hline RW [30] & Random & $x$ & $x$ & $x$ & $x$ & $x$ \\
\hline $\mathrm{RD}[31]$ & Random & $x$ & $x$ & $x$ & $x$ & $x$ \\
\hline MG [32] & Random & $x$ & $x$ & $x$ & $x$ & $x$ \\
\hline GM [35] & Time dependent & $x$ & $\checkmark$ & $\checkmark$ & $x$ & $x$ \\
\hline BSA [32] & Time dependent & $\sqrt{ }$ & $\checkmark$ & $\checkmark$ & $x$ & $x$ \\
\hline GM [35] & Time dependent & $x$ & $\checkmark$ & $\checkmark$ & $x$ & $x$ \\
\hline ST [32] & Time dependent & $\checkmark$ & $x$ & $x$ & $x$ & $x$ \\
\hline SRCM [35] & Path planned & Partially & $x$ & $x$ & $x$ & Partially \\
\hline PPRZM [36] & Path planned & Partially & $x$ & $x$ & $x$ & $x$ \\
\hline CLMN [29] & Group & $x$ & $x$ & $\checkmark$ & $\checkmark$ & Partially \\
\hline NC [32] & Group & $x$ & $x$ & $\checkmark$ & $\checkmark$ & $x$ \\
\hline PRS [32] & Group & $x$ & $x$ & $\checkmark$ & $\checkmark$ & $x$ \\
\hline DPR [7] & Topology control & $\checkmark$ & $x$ & $x$ & $x$ & $x$ \\
\hline SDPC [38] & Topology control & $x$ & $x$ & $x$ & $\checkmark$ & Partially \\
\hline MPB [35] & Time dependent & $x$ & $\checkmark$ & $x$ & $\checkmark$ & $x$ \\
\hline
\end{tabular}

waypoint mobility model, i.e., only focusing UAVs in the center part of simulation area [31]. In this mobility model, each UAV moves towards the edge of the simulation area to select the target, rather than near to the center of simulation area. At the target point, wait until pause time and then select another random target at the edge point of simulation area.

(d) Manhattan grid

The mobility model Manhattan grid (MG) is proposed to describe the movement of UAVs in an urban area using grid road topology [32]. The movements of UAVs in this mobility model are in vertical or horizontal form, where the street layout is very regular. The selection of UAVs movements is based on the probabilistic approach whether to turn right ( 0.25 probability), left ( 0.25 probability), or straight ( 0.5 probability) at an intersection point. It is flexible for the UAVs to change the direction, but this model in not suitable for the most of scenarios of UAV networks due to geographic constraints on UAVs mobility.

2.2.2. Time-Dependent Mobility Models. The time-dependent mobility (TDM) models use different mathematical equations to change the speed and direction smoothly instead of sharp speed and direction changes. The applications of this category are environmental sensing and search and rescue operations. These mobility models are as follows:

(a) Gauss-Markov

Liang and Haas introduced Gauss-Markov (GM) mobility model for the protocol simulation in the wireless ad hoc network [33]. In this mobility model, the speed and direction set first for each UAV and 
then, for each time interval, the movement of UAVs updated accordingly. This model is based on the position, previous speed, and direction of UAVs. GM mobility model is commonly used for simulating the swarm of UAVs behavior in the variable simulation area. Due to high mobility and speed of UAVs, the position of UAVs is dependent on previous position stored in memory [34, 35]. GM mobility model decreases the quick stop and sharp turn as compared to the previous models with the help of related history of speed and direction.

(b) Boundless simulation area

The boundless simulation area mobility model relates the current speed and direction with previous UAVs speed and direction. In this model, the UAVs smoothly change the speed and direction. The UAV moving from one side reaches the target area of simulation and then continues its movement and reappears in the opposite direction of that simulation area. In this mobility model, during simulation, the undesirable effects occur; i.e., the UAVs moving out from an edge and entering from another edge [32]. This mobility model is not able to meet the desired condition due to the teleportation effects inherent to the model where the area of simulation is in two-dimensional form and linkage among prominent mobile UAVs.

(c) Smooth turn

In smooth turn (ST) mobility model, the UAVs select a point in the space and move around it until the UAV chooses another turning point. To ensure smooth movement on the trajectories, the selected point must be perpendicular to the UAV direction. The time spent by the UAV to move around the current turning point is modeled to be exponential distributed. This model allows the mobile UAVs to move freely across spatial and temporal coordinates. This model captures accurately the smooth movement pattern of UAVs without enforcing redundant constraints. The main issue with this model is the lack of method for collision avoidance and the reflection effects from the boundary, i.e., the impact of mobile UAV to enforce for sudden change in its direction on the boundary [32].

2.2.3. Path Planned Mobility Models. The path planned (PP) mobility models work on the preplanned paths to be followed by UAVs during the simulation. Each UAV movement in these models is based on some specific patterns to move from one point to another, and the pattern may be changed or it follows the same pattern [32]. The applications of this category are an agricultural management and traffic and urban monitoring. These models are as follows:

(a) Semirandom circular movement

Semirandom circular movement (SRCM) mobility model is developed for UAV-based ad hoc networks in which the UAV movements are in a circular form or curving manner [35]. In this model during simulation, the UAVs observe the specified region and obtain information about that region. This model is suitable where the UAVs move around a specific region. The mobile UAV moves toward the first destination point on the same circle and waits for pause time, and then it starts again movement towards the second destination point. After completing the rounds in the circle, then it moves towards another circle by selecting it randomly [32]. The flight plan of UAVs is not predetermined but looking for the desired object at different locations in the region.

(b) Paparazzi

The Paparazzi (PPR) mobility model is with a stochastic versatility that imitates properties of paparazzi in the unmanned aerial vehicle system [36]. This model works in light of state machine that has six states of movement pattern, i.e., stay-at, eight, oval, scan, and waypoint. Each UAV selects a movement type, starting position, and speed at the beginning, then for the entire simulation time, it chooses and fixes a random altitude. Due to the lack of managing the movement patterns, the UAV positions are selected randomly. Furthermore, when the UAV completes the first pattern and enters in the second one, it is based on the rotation angle of the scheme [32].

2.2.4. Reference Point Group Mobility Models. Reference point group (RPG) mobility model forms a group of UAVs for movement in a specific domain [35]. The UAVs of a same group move freely in a specific distance with a reference point. Each group reference point is different from one another and can be used to find the position of the member UAVs. This mobility model is commonly used in the clustering approach, in which the reference point known as cluster head and member UAVs means cluster member. The movement of $\mathrm{CH}$ defines the mobility behavior of the entire group. The mobility to each member UAV is assigned with a reference point (i.e., $\mathrm{CH}$ ) that follows the group movement with a predefined path. The member UAVs are randomly placed in cluster range [29]. The applications of this category are environmental sensing and search and rescue operations. These models are as follows:

(a) Column

Sanchez and Manzoni proposed column (CLMN) mobility model for multihop wireless networks where mobile UAVs exchange information with low power, low capacity of transmission channel, and shared medium [37]. The application of this model is to scan or search target UAV in the specified region. The UAVs move around the reference point placed on a predefined line. During the pause time, the new position of each reference point and advance vector of the line is calculated based on the previous position of reference point. Due to the movement of 
UAVs around a specific point with distance and predefined line, this model prevents the collision of UAVs. This model can establish connection-oriented transmission among the UAVs within a same group with the help of spatial constraints. The pitfall is that the UAVs turn smoothly and the random speed changes are not present in this model [32].

(b) Nomadic community

The mobility model nomadic community (NC) is proposed to able the UAVs with random movement around the reference point. The reference point can also move randomly at each time interval. The UAVs in the group form can share the common space as defined by the unique reference point. To avoid the collision among UAVs, the flying space divides managing the distance among UAVs pair. In this mobility model, the unexpected movement may occur because of changes in direction, speed of mobile UAVs, and reference points. The issue is that the UAVs smooth turn and the random speed changes are not present in this model [32].

(c) Pursue

This pursue (PRS) mobility model is similar to the nomadic community model and shares the same features. The mobile UAVs during pursuing the target use a simple random relative motion. In this model, the UAVs follow the particular target that moves in a certain direction. It works like the police officer following and trying to catch the escaped criminals [32].

\subsubsection{Topology Control-Based Mobility Model. Topology} control-based mobility model is the new category to ensure the real-time communication among UAVs during the mission. During the mobility of UAVs, it is a difficult task to maintain connectivity and exchange of information. This mobility model is able to control the network topology by reducing random movement and adding the UAVs aware movement according to the mission. Messous et al. [39] proposed distributed mobility model considering area and connectivity. The following topology control-based mobility model is proposed in which UAV works in a swarm [32]:

(a) Distributed pheromone repel

Kuiper and Nadjm proposed mobility model named distributed pheromone repel (DPR) [7] that defines flexible and random movement of UAVs. Each mobile UAV maintain its own pheromone map; the map has the mobile UAV scan an area in a grid form, where each cell contains a timestamp representing the last time the cell was scanned. The scanned information about the local maps is shared among all the mobile UAVs. The mobile UAVs move according to the direction in map to turn right, left, and go straight. This model provides smooth turns of mobile
UAVs based on the pheromone map. The connectivity issue is not considered in this model.

(b) Self-deployable point coverage

Self-deployable point coverage (SDPC) mobility model is proposed by Sanches-Garcia et al. [38] to cover the mission area and provide connectivity. In this model, a set of UAVs for exchange of information during mission in the disaster area deployed that the victims of the disaster event can use it. The UAVs move around to cover many victims on the ground to maintain connectivity with other UAVs. The simulation of this model is performed with realistic movement of UAVs. The explicit collision detection is not considered in this model.

(c) Mission plan-based

Mission plan-based (MPB) mobility model is designed for UAV-based ad hoc networks [35] in which the UAVs movement plan are predefined in advance. The paths in the mission area are predefined for movement of UAVs each time. The flight time is set for each UAV with speed and direction to get information from the mission area. The mobility or movement information of each UAV is updated time to time; if it reaches the target before the ending time, then it changes the direction to the starting point and continues flight as a round trip.

2.3. Clustering Protocols Based on Swarm Intelligence. The communication of flying nodes (UAVs) is challenging task due to sensitivity, mobility, and dynamic topology of multiple UAVs. Due to the large number of flying nodes, the partition of the nodes into different nonoverlapping clusters becomes an optimization problem. Once the parameters are optimized, it can used for different scenarios. However, to the best of author knowledge, none of the existing techniques utilized a combination of multiple parameters for selection of cluster heads in FASNET. This section will provide a support for the necessity of the FASNET cluster optimization using swarm intelligence. It also explains how to provide optimum cluster organization in order to form balanced cluster with specified node degree, energy, control routing overhead, and provide flexibility with high mobility of nodes [4].

The researchers proposed mobility-aware clustering protocols for FASNET in [40-42] based on weights, dynamic features, and fuzzy logic for FASNET. These protocols have different properties and limitations. The properties of clusters and CHs include size, type of communication (intraor intercluster) of clusters, mobility of $\mathrm{CH}$, type and role of node, load balancing, clustering type, scalability, communication overhead, delay, energy, location awareness, and multihop communication. The limitations are the cluster may be balanced or unbalanced, the communication may be direct or indirect, the nodes may die, the link may down, an interference may occur, and the cluster may be formed with residual energy while ignoring the mobility, degree, and topological changes. 
2.3.1. Bioinspired Mobility Prediction Clustering (BIMPC) Routing Protocol. BIMPC is mobility-aware clustering protocol with mobility prediction using foraging model of Physarum polycephalum [40]. The mobility feature of UAVs integrates with the bioinspired foraging model to solve the conventional clustering issues such as energy utilization of $\mathrm{CH}$, frequent change in topology, and communication overhead. Cluster formation and selection of $\mathrm{CH}$ start during the establishment of UAV networks. All the neighbor nodes broadcast in a range the HELLO packet to make list of neighbors in a table. The UAVs measure the link quality and UAVs availability for $\mathrm{CH}$ selection. Each UAV calculates its probability to be a $\mathrm{CH}$ of the current cluster. After cluster formation, the CM selection is based on the announcement received from the $\mathrm{CH}$ to join us. If the member receives, more than one $\mathrm{CH}$ announcement to join is based on high flexibility in communication. Cluster maintenance is required for solving the issue of high-speed UAVs mobility and link failure. For maintenance of clusters, the BIMPC takes action in four situations: when CM leaves or joins the cluster, when $\mathrm{CH}$ moves around, and when cluster integrates or separates from one another. BIMPC is compared with MOBIC [43], DDVC [44], MPBC [45], and MPCR algorithms [46].

(1) Findings. BIMPC [40] enhances the link and $\mathrm{CH}$ availability in terms of energy to perform stable cluster formation. This algorithm shows stability for dynamic structure of UAV networks with less communication overhead. The mobility considered in bioinspired mobility prediction clustering protocol is moderate, and UAVs in mission-oriented task are with very high mobility and required QoS communication.

2.3.2. Self-Organization-Based Cluster Scheme (SOCS). Khan et al. in [41] proposed a self-organization-based clustering scheme (SOCS) to improve reliable communication among UAVs using glowworm swarm optimization (GSO). The properties of GSO are used to select the optimum route and manage topology. Residual energy, luciferin value, and GS connection of UAVs are considered for the election of $\mathrm{CH}$ and formation of the cluster. The glowworm has its value of luciferin and neighborhood range. The fitness function based on the weights of the above parameters is used for $\mathrm{CH}$ selection. The objective function and its position are depending on the value of luciferin of a glowworm. The UAV with high fitness value considered is $\mathrm{CH}$, and the remaining UAVs become CMs. For the topology management, the position of UAVs is updated using GSO properties. The selection of routes among UAVs is based on residual energy, distance to neighbor, and position of unmanned aerial vehicles. The proposed SOCS performs better in terms of a cluster lifetime, cluster building time, and delivery ratio compared with the existing state of art cluster schemes like grey wolf and ant colony optimization.

(1) Findings. To improve the reliable communication among UAVs, glowworm swarm optimization (GSO) is used for $\mathrm{CH}$ selection and cluster formation based on residual energy, node degree, and distance and position of UAVs [41]. The mention distance of UAVs from $\mathrm{CH}$ is only $5 \mathrm{~m}$. SOCS ignore communication load and control message overhead, which can also reduce the network lifetime. The distance between UAVs and $\mathrm{CH}$ is considered fixed, and the mobility of UAVs is not considered. The mobility of UAVs, speed, and flight time are not mentioned in the article.

\subsubsection{Bioinspired Clustering Scheme for FASNET (BICSF).} Khan et al. in [42] proposed an energy and mobility-aware clustering protocol called BICSF based on glowworm swarm optimization and krill herd (KH). GSO is inspired by the flashing behavior of glowworms. Cluster formation performs based on UAVs residual energy and luciferin value. Fitness function is used to calculate the threshold value; the UAV with the highest value (means high energy and luciferin value) is selected as a $\mathrm{CH}$, and the remaining UAVs become CMs. The flying node with low relative mobility is considered for the selection of $\mathrm{CHs}$. $\mathrm{KH}$ is used to manage and maintain the cluster and CMs movement during the mission. To route information, the selection of optimal route is performed with the help of path detection function. The performance of the proposed protocol is compared with GWO [47] and ACO [48] based on three performance metrics, i.e., cluster formation time, utilization of energy, and network lifetime. The simulation result shows better performance in terms of cluster building time, the lifetime of the network, and energy consumption as compared to GWO and ACO.

(1) Findings. Path detection function is used for routing information because of residual energy, node degree, and distance. The mention distance of UAVs from $\mathrm{CH}$ is only $5 \mathrm{~m}$. BICSF ignores communication load and control message overhead which can also reduce the network life time [42]. The mobility of nodes and environmental changes is considered to provide efficiency, but UAV speed and flight time are not mentioned.

The existing clustering protocols [40-42] are based only on the parameters, as given in Table 2, for the selection of UAV CH while ignoring the location awareness except [41] the communication delay and degree except distance to neighbor [41, 42].

The cluster formation in the existing clustering protocols did not consider the reclustering and balance cluster formation, as shown in Table 3.

The performance metrics of the selected routing protocols are not tested for cluster building time, packet delivery ratio, and cluster lifetime, as shown in Table 4 . In Table 5, the summary of simulation of the existing selected protocols is given with all the considered fields such as mobility model, speed, area of simulation, distance, degree and simulation, and flight time.

\section{Proposed Bee Intelligence Optimization Approach}

The clustering problem in FASNET is formulated to a dynamic optimization problem. The $\mathrm{CH}$ selection and cluster 
TABLE 2: Summary of selected cluster-based routing protocols ( $\mathrm{CH}$ selection parameters).

\begin{tabular}{lcccccc}
\hline Ref. & SIO scheme & Energy & Mobility & Loc. awareness & Deg. & Dist. \\
\hline$[40]$ & {$[19]$} & $\checkmark$ & $\checkmark$ & - & - & - \\
{$[41]$} & {$[20]$} & $\checkmark$ & - & $\checkmark$ & $\checkmark$ & $\checkmark$ \\
{$[42]$} & {$[20]$} & $\checkmark$ & $\checkmark$ & - & $\checkmark$ & $\checkmark$ \\
\hline
\end{tabular}

TABLE 3: Summary of selected routing protocols (cluster formation).

\begin{tabular}{lccccc}
\hline Ref. & CH election & Neighbor criteria & Parameters & Reclustering & Balanced cluster \\
\hline$[40]$ & Distributed & 1-hop & Mobility, energy & Yes & - \\
{$[41]$} & Weighted metric & M-hop & Energy, distance & - & - \\
{$[42]$} & Weighted metric & M-hop & Neighbors, distance & Yes & - \\
\hline
\end{tabular}

TABLE 4: Summary of selected routing protocols (performance metrics).

\begin{tabular}{lccccccc}
\hline Ref. & Comparison scheme & Routing overhead & Delay & Throughput & Delivery ratio & Cluster building time & C. lifetime \\
\hline$[40]$ & {$[43-46]$} & $\checkmark$ & - & - & - & - & $\checkmark$ \\
{$[41]$} & {$[48-51]$} & - & - & - & $\checkmark$ & $\checkmark$ & $\checkmark$ \\
{$[42]$} & {$[47,48]$} & - & - & - & $\checkmark$ & $\checkmark$ & $\checkmark$ \\
\hline
\end{tabular}

TABLE 5: Summary of selected routing protocols (simulation study).

\begin{tabular}{lccccccc}
\hline Ref. & Mobility model & UAV speed & Simulation area & Distance & No. of UAVs & Simul. time & Flight time \\
\hline$[40]$ & - & $40-70 \mathrm{~m} / \mathrm{s}$ & $50 * 50 \mathrm{~km}^{2}$ & - & 100 & - & - \\
{$[41]$} & RPM & - & $1 \times 1,2 \times 2,3 \times 3 \mathrm{~km}^{2}$ & $5 \mathrm{~m}$ & $15,20,25,30,35$ & $120 \mathrm{~s}$ & - \\
{$[42]$} & RPM & - & $1.5 \times 1.5 \mathrm{~km}^{2} 2.5 \times 2.5 \mathrm{~km}^{2}$ & $5 \mathrm{~m}$ & $15,20,25,30,35$ & $120 \mathrm{~s}$ & - \\
\hline
\end{tabular}

formation algorithm are designed based on the objective function obtained as a result of problem formulation. The foraging properties of bee intelligence are used for the $\mathrm{CH}$ selection and cluster formation. The honeybees select food sources with more nectar in an efficient manner. The foraging behavior of honeybees is used to efficiently select the appropriate UAVs as CHs just like the bees select the food sources with more nectar. The cluster maintenance mechanism is developed to accommodate the topology changes in an efficient manner. The efficiency of bee optimization has been tested [52, 53], in similar areas like MANET and WSN, and is found efficient compared to other similar techniques like PPO, GSO, GWO, and ACO. The proposed clustering scheme based on honeybee optimization compared with clustering protocols [40-42] is based on PPO and GSO. The structure of UAV clusters in FASNET is shown in Figure 3.

3.1. Problem Formulation. The modeling of the clustering problem in FASNET is a dynamic optimization problem, and we assume the FASNET to a graph $G(\mathrm{VU}, E)$ that needs to be clustered. Here, VU represents the number of UAVs and $E$ represents the number of communication links between UAVs within the domain.

The clustering problem representation over a number of graphs is actually the identification of $\mathrm{CH}$ sets. We try to keep the same number of UAVs in each $\mathrm{CH}$ sets keeping the size of $\mathrm{CH}$ sets least possible. The structure of UAV clusters in FASNET depicted in Figure 3. The fitness function is based

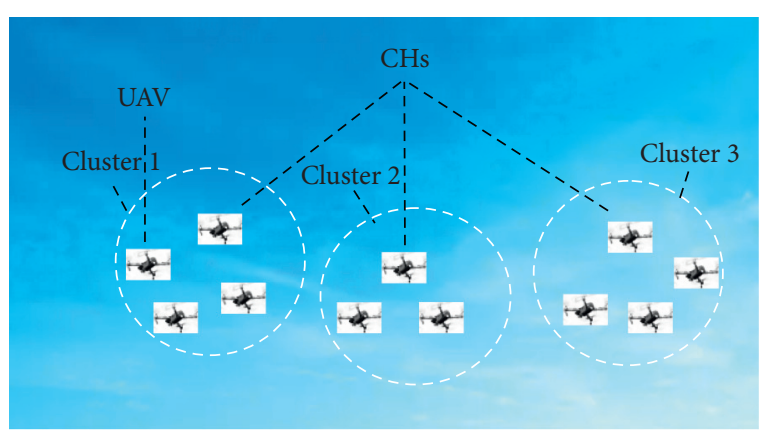

FIGURE 3: Structure of UAV clusters in FASNET.

on the weights to select the UAVs $\mathrm{CH}$ in FASNET as considered in other networks like MANET [54] and WSN [55].

To calculate the fitness of UAVs, the following parameters are considered.

3.1.1. UAV Mobility $\left(M_{U A V}\right)$. In BIMAC, the role of $\mathrm{CH}$ $\mathrm{UAV}$ is assigned to UAV based on the combined weights of different parameters. Mobility is one of the important parameters to consider during the $\mathrm{CH}-\mathrm{UAV}$ selection process. The mobility may cause unstable clusters due to the regular link update of UAVs. Hence, the mobility of UAV needs special attention to achieve stable and long-life clusters. The UAV transmits signals in a circular area with radius $r$. The area contains say " $k$ " UAVs. The UAVs with the 
transmission range may be closer or far. In BIMAC, the transmission zone of UAV is divided into a safe zone and danger zone [56]. As shown in Figure 4, the inner circle with distance $\beta 1 d$ forms the safe zone, while the zone with width $(\beta 2-\beta 1) d$ forms the danger zone. The coefficients $\beta 2$ and $\beta 1$ are selected on the basis of UAVs mobility in FASNET.

The UAV suitable to perform the duties of $\mathrm{CH}-\mathrm{UAV}$ is selected based on UAV relative mobility. The relative mobility is computed by using the signal strength of UAVs. The success of hello packets between sender UAV and receiver UAV represents its distance. To calculate the relative mobility of $\mathrm{UAV} i$ and $\mathrm{UAV} j$, the following equation is used:

$$
M_{\mathrm{UAV}_{i}}\left(\mathrm{UAV}_{j}\right)=10 \log _{10} \frac{R_{\mathrm{ec}} P_{\mathrm{wrUAV}_{j}}^{n} \longrightarrow \mathrm{UAV}_{i}}{R_{\mathrm{ec}} P_{\mathrm{wrUAV}_{j}}^{o} \longrightarrow \mathrm{UAV}_{i}},
$$

where $R_{\mathrm{ec}} P_{\mathrm{wrUAV}_{j}}^{n} \longrightarrow \mathrm{UAV}_{i}$ is the new receiving power of UAVs from $\mathrm{UAV}_{j}^{j}$ to $\mathrm{UAV}_{i}$, while $R_{\mathrm{ec}} P_{\mathrm{wrUAV}_{j}}^{o} \longrightarrow \mathrm{UAV}_{i}$ is the old receiving power of the Hello packet from $U_{A} V_{j}$ to $\mathrm{UAV}_{i}$. If the value of the above equation is negative, this shows that $\mathrm{UAV}_{j}$ is moving away from $\mathrm{UAV}_{i}$, and vice versa.

For each nearby $U A V_{j}$ of $\mathrm{UAV}_{i}$, we find its range predictor $R_{\text {pre }}\left(\mathrm{UAV}_{j}, \mathrm{UAV}_{i}\right)$ w.r.t. $\mathrm{UAV}_{i}$. We categorize the UAVs based on the space among the UAVs and their relative mobility, as shown in equation (2). A UAV lying in the danger zone and the relative movement is less than zero; it means that the mobility UAV is in the opposite direction. In the $\mathrm{CH}-\mathrm{UAV}$ selection process, these UAVs will be kicked out from the competition of CH-UAVs. The weight of these

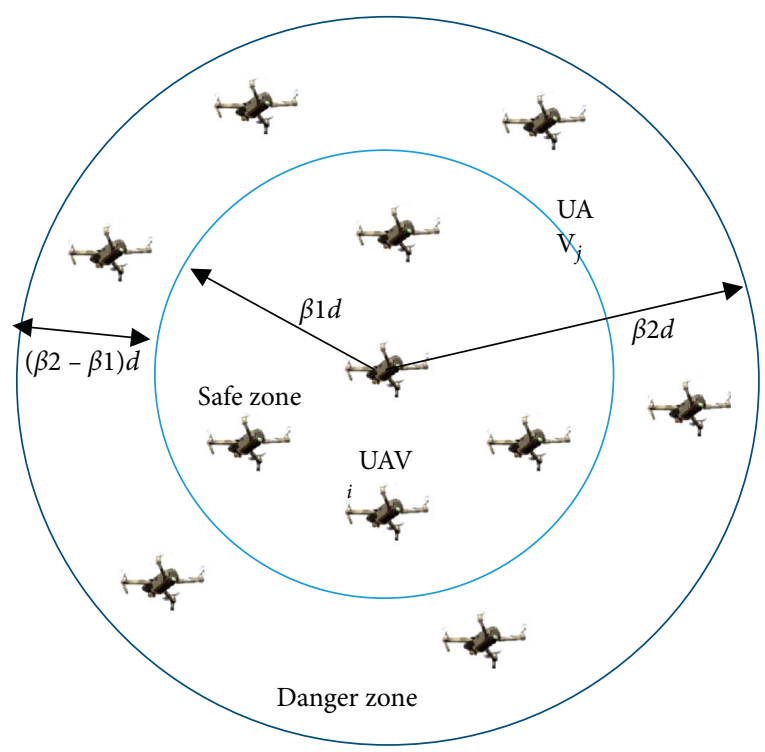

Figure 4: Transmission zones.

nodes will also be negative, i.e., “-1." Similarly, for the UAV having a relative mobility value greater than zero and lying within the transmission range, it means that the UAV is getting closer and its weight will be "1." For the UAVs within the safe zone but moving in the opposite direction, the weight "0" will be assigned to these UAVs, as shown in the following equation:

$$
R_{\text {pre }}\left(\mathrm{UAV}_{j}, \mathrm{UAV}_{i}\right)=\left\{\begin{array}{l}
0, \\
1, \\
1+\frac{\operatorname{dis}\left(\mathrm{UAV}_{i}, \mathrm{UAV}_{j}\right)-\beta 1 d,}{(\beta 2-\beta 1) d}
\end{array}\right.
$$

if $\beta 1 d<\operatorname{dis}\left(\mathrm{UAV}_{i}, \mathrm{UAV}_{j}\right)<=\beta 2 d \wedge M_{\mathrm{UAV}_{i}}\left(\mathrm{UAV}_{j}\right)<0$,

if $\operatorname{dis}\left(\mathrm{UAV}_{i}, \mathrm{UAV}_{j}\right)<=d \wedge M_{\mathrm{UAV}_{i}}\left(\mathrm{UAV}_{j}\right)>0$,

if $\operatorname{dis}\left(\mathrm{UAV}_{i}, \mathrm{UAV}_{j}\right)<=\beta 1 d \wedge M_{\mathrm{UAV}_{i}}\left(\mathrm{UAV}_{j}\right)<0$.

3.1.2. UAV Degree $\left(D_{U A V}\right)$. In the proposed BIMAC, the extra significance is set to the choice of network parameters. Parameters have a strong impact on the network lifetime, cluster lifetime, load balancing, and reaffiliation. The number of UAVs in FASNET plays a significant role because it ensures that the lowest number of UAVs is required to cover the targeted region. To calculate the minimum number of UAVs required to cover a certain region, the projected area and the transmission range of UAVs are required. This can be achieved by dividing the total area by the area of the hexagon. The BIMAC will manage the overlapping clusters because the hexagon is considered instead of a circle for easy calculations:

$$
A_{\mathrm{FASNET}}=W \times L\left(m^{2}\right),
$$

where $A_{\mathrm{FASNET}}$ is the targeted region and $W$ and $L$ are the width and length of the targeted field. The coverage region of UAV can be calculated as

$$
A_{\mathrm{UAV}}=3 \frac{\sqrt[3]{2}}{2} a^{2}
$$

The minimum number of UAVs $\left(\mathrm{UAV}_{m}\right)$ to fully cover the targeted region can be obtained by the following equation:

$$
\mathrm{UAV}_{m}=\frac{A_{\mathrm{FASNET}}}{A_{\mathrm{UAV}}}
$$

Here, $A_{\text {FASNET }}$ is the targeted region of the FASNET and $A_{\mathrm{UAV}}$ is the area covered by a single $\mathrm{UAV}$ in regular 
hexagon. The number of optimal UAVs in a cluster can be calculated by the following equation:

$$
\mathrm{UAV}_{O}=\frac{\mathrm{UAV}_{n}}{\mathrm{UAV}_{m}} .
$$

In the equation above, $\mathrm{UAV}_{n}$ is the total number of UAVs in the FASNET and $\mathrm{UAV}_{m}$ is the minimum number of UAVs to fully cover the targeted region.

Hence, the projected number of neighbors of a node in the FASNET can be obtained by the following equation:

$$
\mathrm{UAV}_{p}=\frac{\mathrm{UAV}_{c} \bmod \left(\mathrm{UAV}_{o}+1\right)}{\mathrm{UAV}_{o}},
$$

where $\mathrm{UAV}_{c}$ denotes the total number of current neighbors.

The UAV having maximum neighbors is the best candidate to become a $\mathrm{CH}-\mathrm{UAV}$.

Here, $\mathrm{PD}_{i}$ is the probability with respect to UAV degree and is calculated as

$$
\mathrm{PD}_{i}=C_{p}+\frac{\mathrm{UAV}_{p}}{\mathrm{UAV}_{n}},
$$

where $C_{p}$ is the percentage of CH-UAVs, $\mathrm{UAV}_{p}$ is the projected number of neighbors, and $\mathrm{UAV}_{n}$ is the total number of UAVs in the FASNET.

3.1.3. UAV Energy $\left(E_{U A V}\right)$. The UAV with high energy is the most appropriate candidate for the role of UAVs $\mathrm{CH}$. The UAV with low energy has minimum chances or no probability to become a $\mathrm{CH}$. The percentage of $\mathrm{CH}-\mathrm{UAVs}\left(C_{p}\right)$ is set initially (say 10\%) because the optimal number of $\mathrm{CH}$ UAVs cannot be determined in advance. To limit the initial $\mathrm{CH}-\mathrm{UAV}$ announcements, $C_{p}$ is used in the proposed BIMAC scheme. It has no direct influence on the final $\mathrm{CH}$ UAVs. Prior to the execution of BIMAC, the probability of $\mathrm{CH}-\mathrm{UAV}$ with respect to UAV energy is calculated as

$$
\mathrm{EP}_{i}=C_{P}+\frac{E_{R}}{E_{M}},
$$

where $E_{R}$ is the projected residual energy of $\mathrm{UAV}_{i}$ and $E_{M}$ is the maximum energy of UAV in FASNET.

Fourth, the number of clusters calculated before selecting the $\mathrm{CH}$ for UAVs is taken. After the computation of all UAVs weight, the fitness of CH-UAVs set is calculated by a minimization function. The minimization function used in the proposed BIMAC is discussed in the next section.

3.1.4. Combined Weights. The following equation is used to compute the weight of $\mathrm{UAV}_{i}$ to play the role of $\mathrm{CH}-\mathrm{UAV}$ :

$$
W_{\mathrm{UAV}_{i}}=M_{\mathrm{UAV}_{i}}+\mathrm{PD}_{\mathrm{UAV}_{i}}+\mathrm{EP}_{\mathrm{UAV}_{i}} .
$$

The number of clusters $k$ must be computed prior to choosing the UAVs $\mathrm{CH}$ set by the equation. The value of $\mathrm{K}$ is obtained as

$$
k=\operatorname{round}\left(\frac{1}{n} \sum_{i=1}^{n} \operatorname{Deg}_{i}\right)+1 .
$$

The number of CH-UAVs in FASNET is represented by $k, n$ is the number of UAVs in FASNET, and Deg $_{i}$ represents the direct neighbors of $\mathrm{UAV}_{i}$.

The UAVs-CH set comprises UAVs no less than 3-hop distance. After the computation of all UAVs weight, the fitness of UAVs-CH set is tested by the minimization function (Algorithms 1 and 2).

3.2. BIMAC Algorithm. The notations used in BIMAC algorithm are given in Table 6.

The proposed solution is validated via a series of simulation experiments, and the results are compared with [40-42] clustering protocols based on PPO and GSO in FASNET. The advantages of this optimization technique are simplicity, flexibility, exploring local solutions, managing objective cost, easy implementation, and solving complex functionalities.

\section{Bioinspired Mobility-Aware Clustering Scheme Based on Honey Bee Foraging Behavior}

Clustering schemes in FASNET based on bee intelligence refer how to form and maintain mobility-aware balanced clusters and how to select optimum $\mathrm{CH}$ s in each cluster. Due to the large number of flying UAVs, the partition of the UAVs into diverse nonintersecting clusters becomes an optimization issue [4].

The focus in this section is on cluster-based routing with optimization schemes using bee intelligence showing how to provide optimum cluster organization in order to form a balanced cluster with dynamic node degree, control communication load, and provide flexibility with a high mobility of UAVs.

The frequent change in the FASNET topology brings an additional challenge of mobility. Due to the autonomous system in most scenarios, the path selection is based on the previous speed and direction. In flying ad hoc networks, the random waypoint mobility model is used in which flexibility for paths selection is favored to flying UAVs. The bioinspired mobility-aware clustering scheme having a low reaffiliation rate is considered better among others. The mobility during $\mathrm{CH}$ selection and cluster formation is considered.

4.1. UAV CH Selection. The flying sensor nodes mobility in FASNET is very high compared to other ad hoc networks [57]. Optimal CHs are selected if the reaffiliation rate is very low. The topology variations are mainly because of UAV movements. The consideration of relative mobility during the $\mathrm{CH}$ selection process results in stable clusters and the reclustering procedure called less frequently. The parameters 


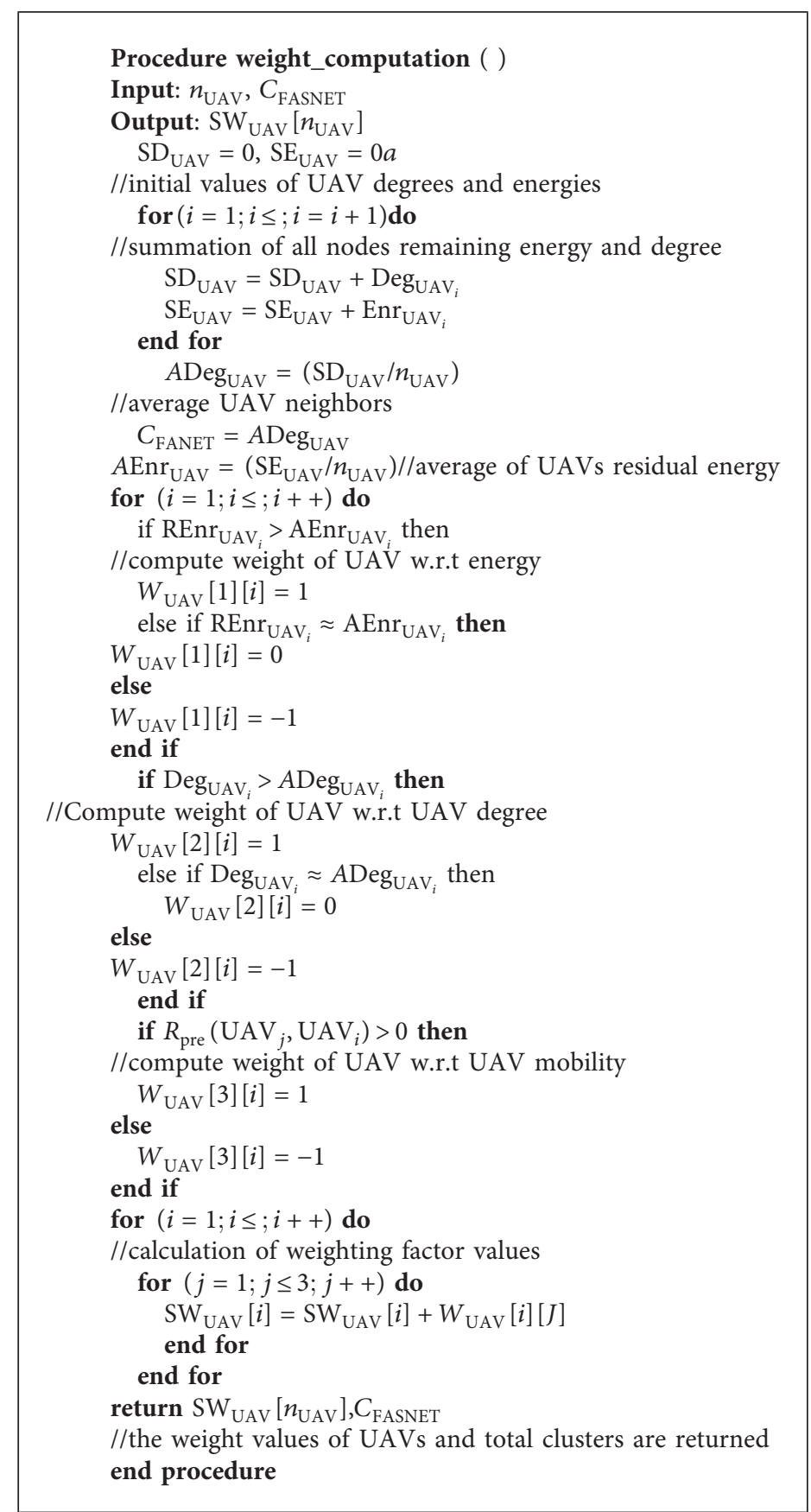

Algorithm 1: Psuedocode of UAV weight computation.

like energy, mobility, location awareness, communication delay, degree, and distance, as shown in Table 2, must be keep in mind during the selection process of mobility-aware CHs.

To select the CH-UAVs in BIMAC, a random set of UAVs is selected initially, as shown in the flow chart in Figure 5.

The fitness of the solution is evaluated on the basis of the following equation:

$$
\begin{aligned}
& \text { Minimize }- \text { Function }\left(W_{\mathrm{UAV}}, \mathrm{AFV}\right) \\
& =\sum_{i=1}^{n} \sum_{j=1}^{k} \mathrm{RW}_{\mathrm{UAV}_{i j}}\left(W_{\mathrm{UAV}_{i}}-\mathrm{AFV}_{j}\right)^{2},
\end{aligned}
$$

subject to $\in \mathrm{RW}_{\mathrm{UAV}_{j}}=1(j=1,2,3, \ldots, k)$ and $\mathrm{RW}_{\mathrm{UAV}_{i j}}$ $=0$ or $1(i=1,2,3, \ldots, n / j=1,2,3, \ldots, k)$

Here, $n$ represent the total UAVs in FASNET, $k$ is the total CH-UAVs, $\mathrm{RW}_{\mathrm{UAV}_{i j}}$ is the relation of $\mathrm{UAV}_{i}$ with cluster 


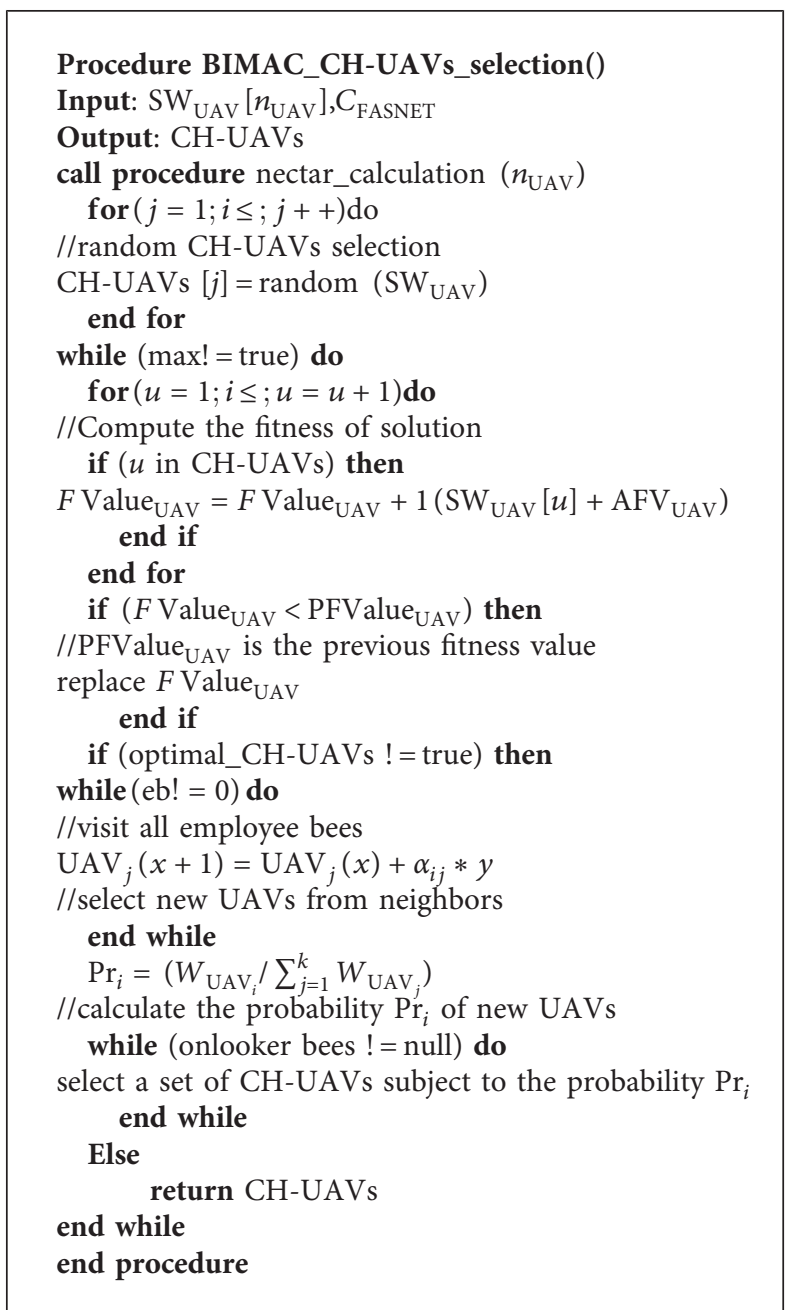

Algorithm 2: Psuedocode of bioinspired mobility-based clustering (BIMAC).

TABLE 6: Algorithm notations.

\begin{tabular}{lc}
\hline Symbols & Definition \\
\hline$n_{\mathrm{UAV}}$ & Total UAVs in FASNET \\
$C_{\mathrm{FANET}}$ & Clusters in FASNET \\
$\mathrm{UAV}_{p}$ & Projected/average value of UAV degree \\
$\mathrm{UAV}[n]$ & Array of UAV IDs \\
$\mathrm{FV}\left(W_{\mathrm{UAV}}, \mathrm{AFV}\right)$ & Fitness value of CH set \\
$\mathrm{SD}_{\mathrm{UAV}}$ & Sum of all UAVs degrees \\
$\mathrm{SE}_{\mathrm{UAV}}$ & Sum of all UAVs energy \\
$\mathrm{Deg}_{\mathrm{UAV}}$ & Degree of UAV \\
$\mathrm{CHs}_{i}$ & Cluster heads \\
$W_{\mathrm{UAV}}[3]\left[n_{U A V}\right]$ & Vector of UAV weights \\
$\mathrm{SW}_{\mathrm{UAV}}$ & Sum of UAV weights \\
$\mathrm{eb}$ & Employee bee \\
$\alpha_{i j}$ & Patch size \\
$z$ & Random variable $[z=-1 \mid 1]$ \\
$\operatorname{Pr}_{i}$ & Probability of UAV \\
\hline
\end{tabular}

$j, W_{\mathrm{UAV}_{i}}$ is the weight of $\mathrm{UAV}_{i}$, and $\mathrm{AFV}_{j}$ is the average fitness of UAV to perform the role a $\mathrm{CH}-\mathrm{UAV}$. The value of $\mathrm{AFV}_{j}$ can be calculated as follows:

$$
\mathrm{AFV}_{j}=\frac{1}{n_{j}} \sum_{j=1}^{k} \mathrm{RW}_{\mathrm{UAV}_{i j}} W_{\mathrm{UAV}_{i}},
$$

where $n_{j}$ represent the total number of UAVs in the $j^{\text {th }}$ cluster and $\mathrm{RW}_{\mathrm{UAV}_{i j}}$ represents the relation of UAV with this cluster $j$. Its value will be 1 if the UAV is a member of the cluster, or it would be 0 if the condition is not true.

After the completion of the initial phase, the local search of bees will be initiated once the optimal set of CH-UAVs has not been found. The scout bees perform the local search in the neighborhood and proposed new solutions. Hence, the employed bee's memory will be updated based on available information either visual or local. The new information will be overwritten on the old information of the employed bee's memory. The memory may be updated on the basis of tests carried out via equation (10) (used for the calculation of nectar volume) to get new solutions.

In this scenario, if the volume of $\mathrm{CH}-\mathrm{UAV}$ s previous nectar is greater than volume of $\mathrm{CH}-\mathrm{UAV}$ new calculated nectar, then the bee maintains the previous information in her memory. If the volume of the new nectar is grater, the 


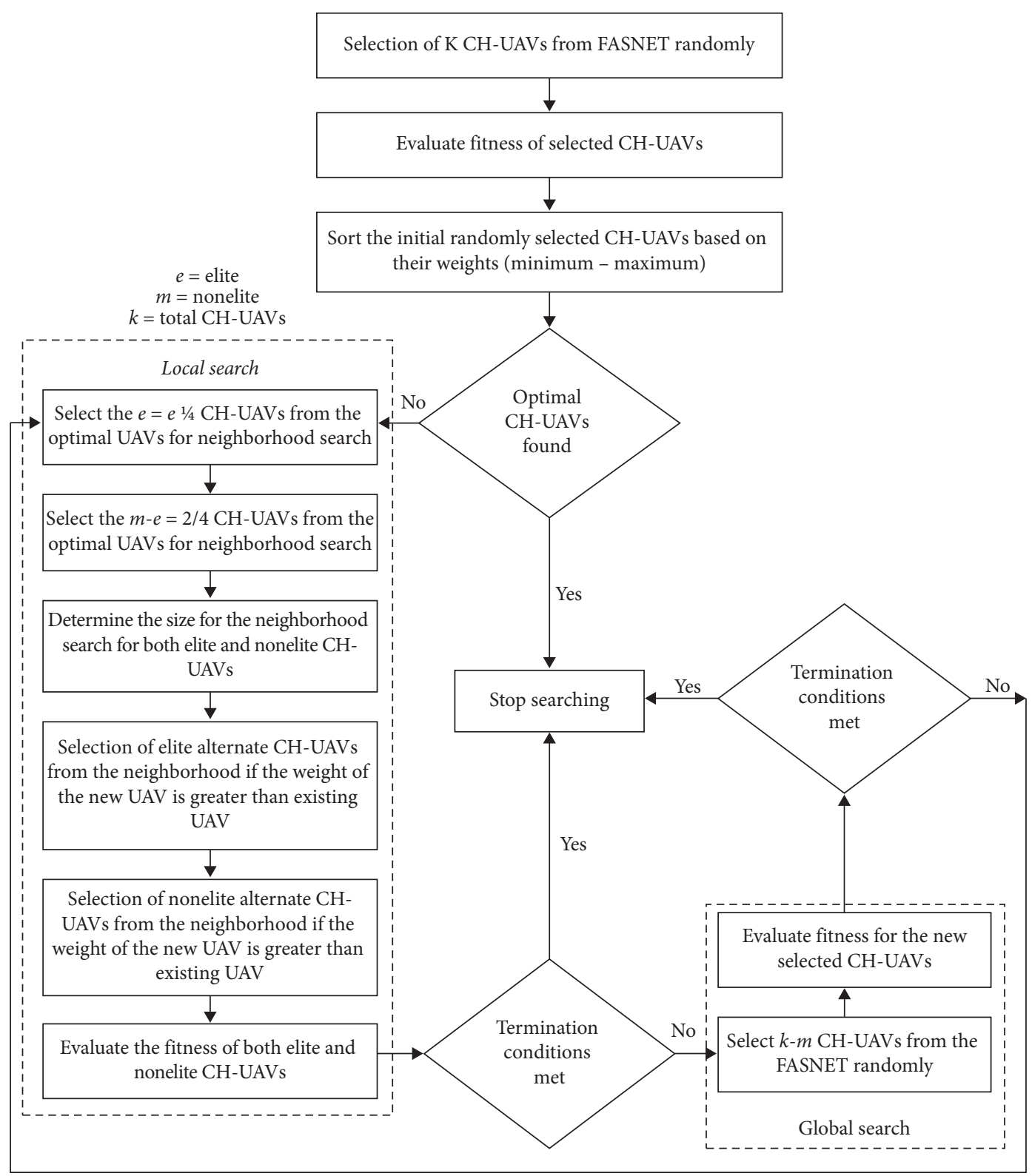

Figure 5: Flowchart of BIMAC algorithm.

bee remembers the novel nectar volume and disremembers the earlier (stowed in bee's memory).

When the employed bee arrives to the hive after completing the search process, it performs a special type of dance on the dancing floor. The dance is performed to communicate the information of $\mathrm{CH}-\mathrm{UAV}$ s nectar volume and direction. The direction of dance also shows the food source direction. The dance may be performed on a different pattern to communicate different types of information with other bees.

The onlooker bees watch the dance performance of employed bees on the dance floor in a careful way to find the direction and volume of nectar.

The novel CH-UAV will be chosen on the basis of their possibility associated with the volume of nectar $W_{\mathrm{UAV}}$. The onlooker bees watch the dance performance of employed bees on the dance floor and decide to visit the $\mathrm{CH}-\mathrm{UAV}_{j}$ with probability $\mathrm{pr}_{i}$ :

$$
\operatorname{Pr}_{i}=\frac{W_{\mathrm{UAV}_{i}}}{\sum_{j=1}^{k} W_{\mathrm{UAV}_{\mathrm{j}}}},
$$

where $W_{\mathrm{UAV}_{i}}$ is the volume of nectar at $\mathrm{UAV}_{i}$, and the onlooker discovers the neighborhood spot or nectar location $(\mathrm{CH}-\mathrm{UAV})$ in the radius of $\mathrm{UAV}_{j}$ by using the following equation:

$$
\mathrm{UAV}_{j}(x+1)=\mathrm{UAV}_{j}(x)+\alpha_{i j} * y .
$$

In the equation above, the patch size to search neighbors is $\alpha_{i j}$ and var represents a uniform random variable. The value of var lies in the range $[-1,1]$. 
4.2. UAV Cluster Formation. Once the $\mathrm{CHs}$ are selected, the result will be stable cluster formation. To minimize the reclustering for the formation of stable clusters, relative UAVs mobility is considered during $\mathrm{CHs}$ selection. The $\mathrm{CH}$ election, neighbor criteria, and clustering parameters must be kept in mind during the process of balanced cluster formation. The summary is given in Table 3 .

Once the $\mathrm{CH}-\mathrm{UAV}$ s are selected, it broadcasts a message in the FASNET. The message contains information about their location, status, and ID. The UAVs that receive the message will join the cluster. The cluster member communicates this information with $\mathrm{CH}-\mathrm{UAV}$. If a UAV receives the membership message from more than one $\mathrm{CH}$ UAV, the UAV will join the nearest CH-UAV. In case of tie, the UAV will choose randomly to join a $\mathrm{CH}-\mathrm{UAV}$.

4.3. Performance Evaluation and Simulation Study. This section provides simulation study and performance evaluation of the proposed bioinspired mobility-aware clustering (BIMAC) algorithm compared for the first time with the existing UAVs clustering algorithms BIMPC [40], SOCS [41], and BICSF [42]. The BIMPC [40] uses Physarum polycephalum optimization (PPO) and only considers mobility as the criteria for $\mathrm{CH}$ selection. The SOCS [41] uses glowworm swarm optimization (GSO) and considers the residual energy, location awareness, and connectivity for the $\mathrm{CH}$ selection and balance cluster formation. The BICSF [42] also uses glowworm swarm optimization (GSO) and considers residual energy, neighbor UAVs range, and location awareness as the criteria for the $\mathrm{CHs}$ selection. The proposed BIMAC algorithm uses honeybees foraging optimization. Multiple parameters are considered, i.e., residual energy, mobility, degree, and communication load of UAVs for optimum $\mathrm{CH}$ selection and balanced cluster formation that will enhance the network lifetime with high mobility of UAVs. The simulation tool is EstiNet. The evaluation metric used in our simulation is: average link-connection lifetime, $\mathrm{CH}$ lifetime, member UAVs per cluster, reassociation time, and cluster formation time. IEEE 802.11 standard air interface is selected for GS communication to UAVs, while IEEE 602.16 is used to extend the coverage up to $50 \mathrm{~km}$ for UAVs-to-UAVs or to BS communication [58]. The rest of parameters are given in Table 7.

The connection between the $\mathrm{CH}$ and its member UAVs play a vital role due to the mobility of UAVs and communication range. The UAV's intracluster and intercluster fast movement made frequent changes in the topological structure. The stable link connection for a long time increases the overall network lifetime and the performance of the algorithm. The simulation result in Figure 6 represents the UAV movement and the average link connection of the BIMAC, BIMPC [40], SOCS [41], and BICSF [42]. The range of communication for the UAVs considered $10 \mathrm{~km}$ and speed $55 \mathrm{~m} / \mathrm{s}$. The performance index shows the average time for the established connection between the member UAVs with its $\mathrm{CH}$. The proposed BIMAC algorithm average link connection duration is greater than [40-42] that with the change in the speed of UAVs towards the maximum limit.
TABLE 7: Simulation parameters.

\begin{tabular}{lc}
\hline Parameters & Values \\
\hline Field size & $50 * 50 \mathrm{~km}^{2}$ \\
No. of UAVs & 100 \\
Distance among UAVs & $10 \mathrm{~m}$ \\
Transmission range & $10 \mathrm{~km}-20 \mathrm{~km}$ \\
Standard with MAC & IEEE $802.11 \& 802.16$ \\
Spectrum & $2.3-2.5 \mathrm{GHz}$ and $3.4-3.5 \mathrm{GHz}$ \\
Mobility model & RWP model in Section 2 \\
Speed of UAVs & $40 \mathrm{~m} / \mathrm{s}-70 \mathrm{~m} / \mathrm{s}$ \\
UAVs location strategy & Random placement \\
\hline
\end{tabular}

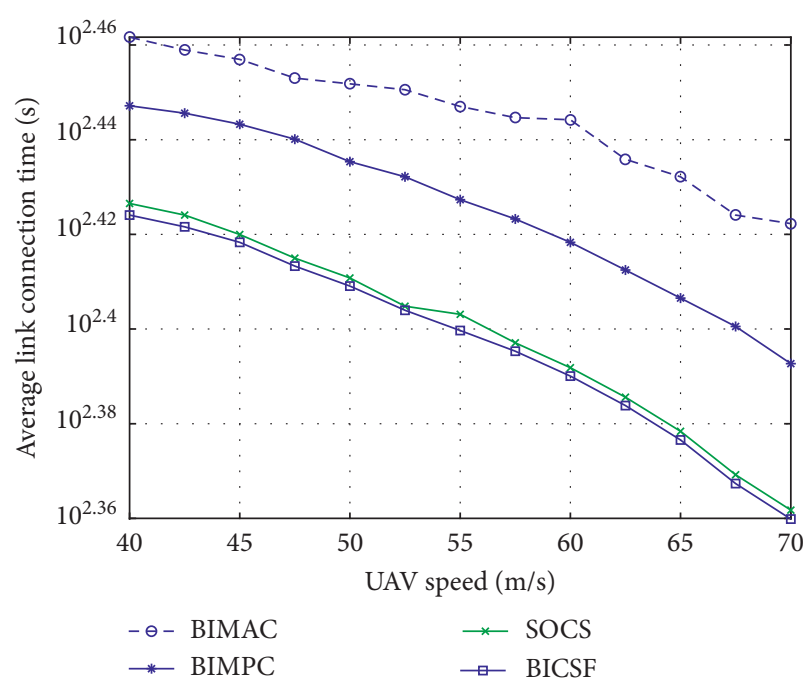

Figure 6: Average link connection lifetime vs UAV speed.

The balanced cluster formation process based on constraints of the BIMAC algorithm improves the stability of $\mathrm{CH}$ and its member UAVs. This shows that BIMAC is more suitable for dynamic networks. The algorithm [40-42] performance decreases with the variation towards the maximum speed of UAVs. The increase in UAVs speed also results in the frequent variation in the topological structure of UAVs network. For that reason, in general, the lifetime of the average link connection reduces gradually. The simulation result also shows that the BIMPC algorithm [40] considers mobility during the cluster formation and $\mathrm{CH}$ selection; that is why the average link connection lifetime reduces gradually compared to the other two algorithms [41, 42].

The duration of link connection is also dependent on the UAVs range of communication. The UAVs' movement outside of the $\mathrm{CH}$ communication range decreases the probability of the link connection lifetime with its $\mathrm{CH}$, which also degrades the network lifetime. The simulation result in Figure 7 represents the communication range and the average link connection lifetime of the BIMAC, BIMPC [40], SOCS [41], and BICSF [42]. The UAVs speed considered $55 \mathrm{~m} / \mathrm{s}$, and range of communication is $10 \mathrm{~km}$. The BIMAC and other algorithms average link-connection lifetime enhances by the movement of UAVs towards maximum communication range. BIMAC algorithm shows better 


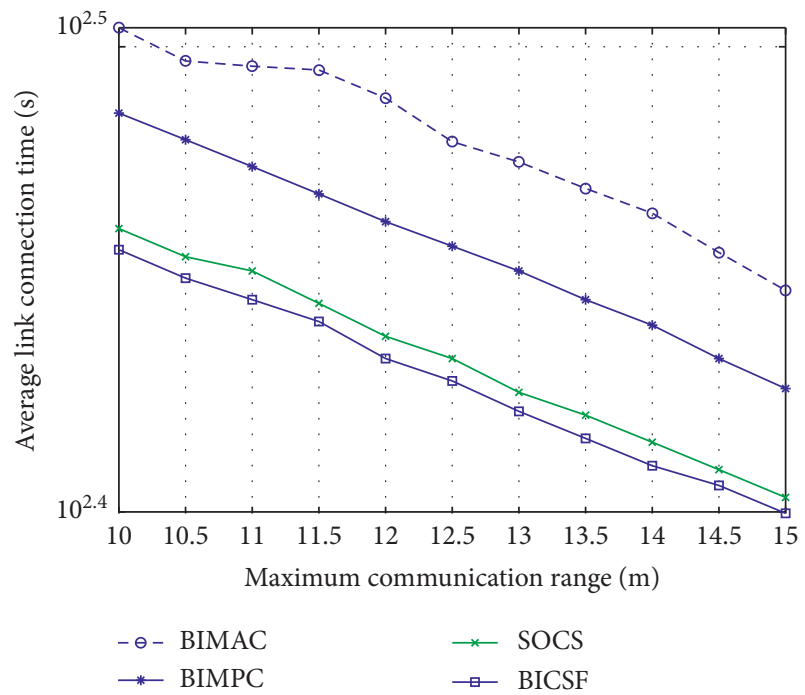

Figure 7: Average link connection lifetime vs maximum communication range.

performance results in average link connection lifetime among the selected algorithms [40-42].

The proposed BIMAC is based on honeybees foraging model and considering the UAVs mobility, energy, degree, and communication load for selecting the optimum $\mathrm{CH}$. Thus, the efficient mechanism for selecting the $\mathrm{CH}$ increases the $\mathrm{CH}$ lifetime with high mobile UAVs. The $\mathrm{CH}$ s in BIMAC algorithm have more stability compared to other algorithms. The simulation result in Figure 8 represents the UAVs speed and the average $\mathrm{CH}$ lifetime of the BIMAC, BIMPC [40], SOCS [41], and BICSF [42]. The UAVs speed considered $55 \mathrm{~m} / \mathrm{s}$, and range of communication is $10 \mathrm{~km}$. The UAVs fast movement towards the maximum limit made frequent changes in the topological structure, and the $\mathrm{CH}$ rotation is more dramatic. The simulation results show that, with the increase in UAVs speed, all the algorithms average $\mathrm{CH}$ lifetime decreases gradually.

The simulation result in Figure 9 represents the maximum communication range and the average $\mathrm{CH}$ lifetime for BIMAC and algorithms in [40-42]. The BIMAC and other algorithms average $\mathrm{CH}$ lifetime increases by the movement of UAVs' towards the maximum communication range. The average $\mathrm{CH}$ duration degrades with the UAVs movement towards the maximum transmission range. UAV's high mobility has a direct impact on the increase in communication range that affects the stability of $\mathrm{CH}$. BIMAC algorithm shows better efficiency among all other selected existing algorithms in terms of average $\mathrm{CH}$ lifetime.

The number of UAVs in each cluster (UAV degree) should be approximately the same or minimum in order to balance the load on each $\mathrm{CH}$. The degree of UAVs during balance cluster formation plays a vital role. The simulation result in Figure 10 represents the UAV speed maximum limit and the UAV degree for the proposed algorithms BIMAC, BIMPC [40], SOCS [41], and BICSF [42]. The UAV speed considered is $55 \mathrm{~m} / \mathrm{s}$. It does not matter how the changes occur in UAV speed; the UAV degree of $[41,42]$ is

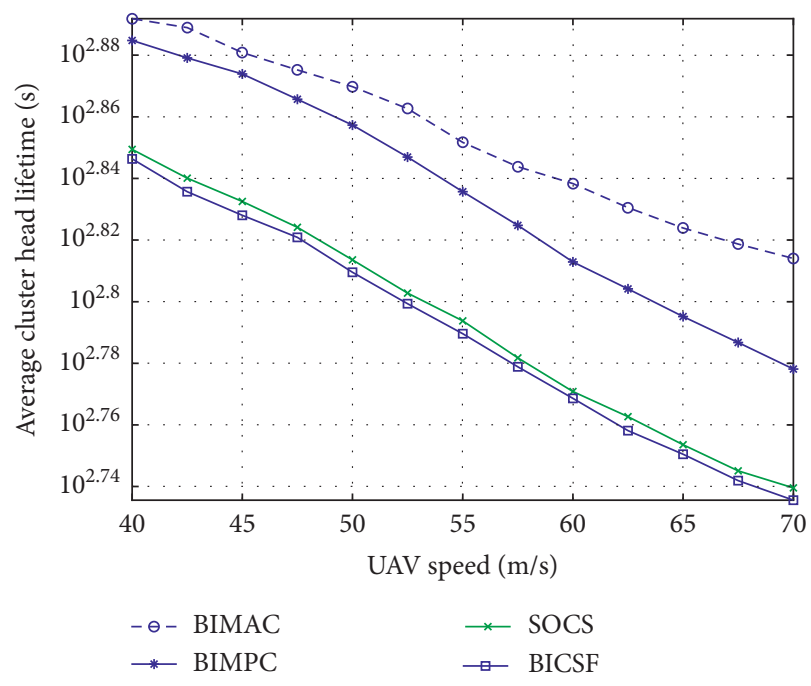

Figure 8: Average $\mathrm{CH}$ lifetime vs UAV speed.

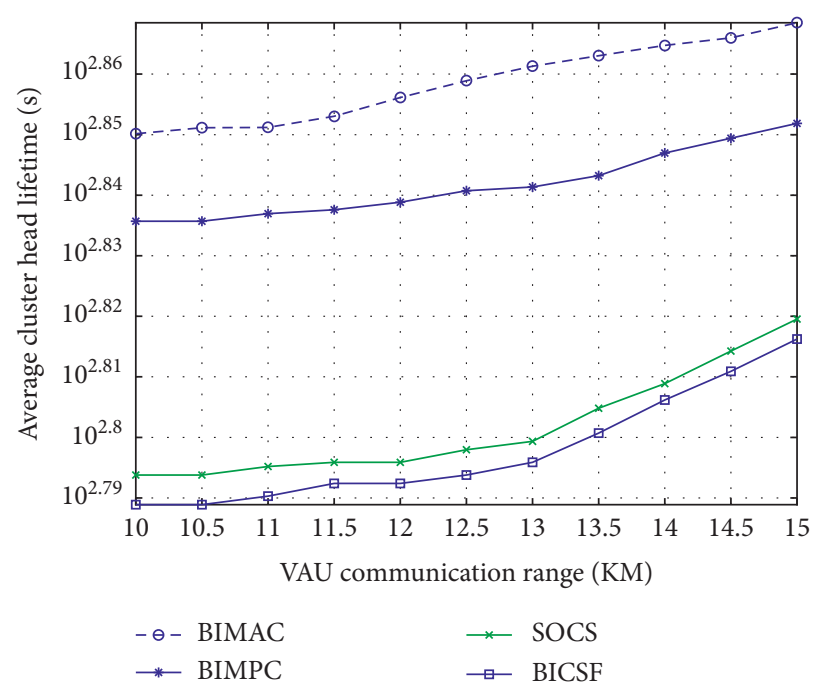

Figure 9: Average $\mathrm{CH}$ lifetime versus UAVs communication range.

almost the same per cluster. The UAV fast movement towards the maximum limit enhances the probability of member UAVs to escape from the communication range, and thus, the UAV degree will reduce gradually. The entire proposed algorithms during simulation show a negative association between the UAV speed and degree. The degree of UAVs in BIMAC is less than the other selected algorithms considering the multiple parameters for cluster membership of UAVs. Only those UAVs will be considered as a member of a cluster that fulfills the criteria to join or leave the cluster. BIMAC algorithm takes care of the member UAVs leaving the current cluster and removes its status to form a stable cluster, but in other selected algorithms, the member UAVs still belong to the current cluster. BIMAC algorithm also tries to minimize the reclustering that results in stable clustering. 


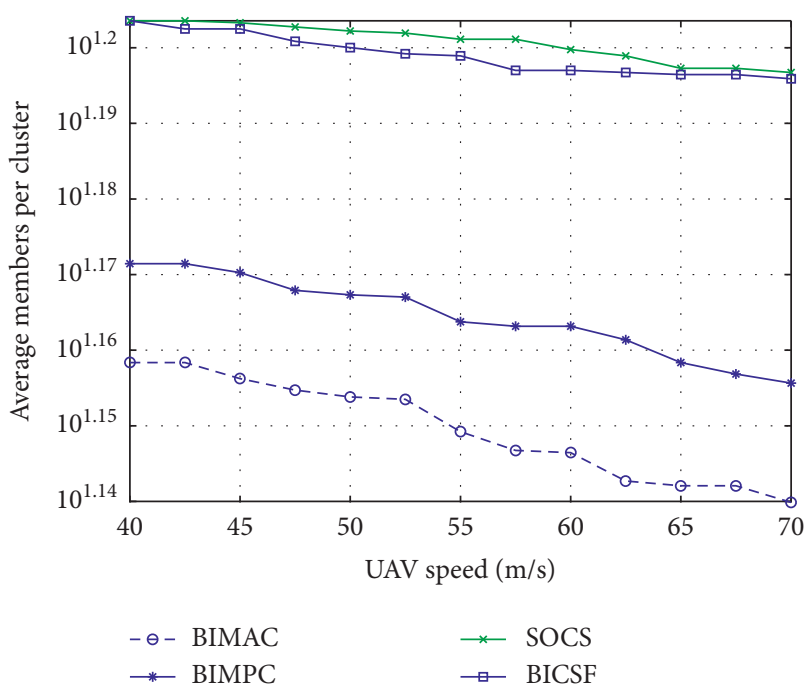

Figure 10: UAVs degree versus UAV speed.

The simulation result in Figure 11 represents the maximum communication range and the average member per cluster for the proposed algorithms BIMAC and that in [40-42]. The communication range is considered $10 \mathrm{~km}$. It does not matter how the changes occur in UAV communication range, and the UAV degree of $[41,42]$ is almost the same per cluster. The BIMAC and all the selected algorithms [40-42] during simulation show that the expansion of transmission range in the degree of UAVs gradually increases. The BIMAC algorithm has still cared to keep the degree of UAVs lower, as shown in Figure 11.

The optimal $\mathrm{CH}$ selection is based on the very low reaffiliation rate. The changes in the topology are mainly due to the UAV speed. The multiple parameters, i.e., mobility, energy, degree, and communication load, are considered during the selection process of high mobility-aware $\mathrm{CH}$. The simulation result in Figure 12 represents the UAV speed and the average reassociation time for the proposed BIMAC, BIMPC [40], SOCS [41], and BICSF [42] algorithms. The UAV speed considered is $55 \mathrm{~m} / \mathrm{s}$. With the increase in UAV speed towards the maximum limit, the proposed BIMAC algorithm has still minimum average reassociation time than other selected algorithms in [40,41] and [42]. The reason is the use of multiple parameters in BIMAC, as mentioned prior in this section.

The simulation result in Figure 13 represents the average reaffiliation time and communication range for the BIMAC, BIMPC [40], SOCS [41], and BICSF [42] algorithms. The communication range is considered $10 \mathrm{~km}$. The probability of reaffiliation time with high-speed UAVs to its cluster as a member increases with the enhancement of communication range towards the maximum limit, but in the simulation results with the increase in communication range, the average reaffiliation time reduced. The proposed BIMAC algorithm has still less average reaffiliation time among all the selected algorithms.

The foraging behavior of honeybees used for cluster formation considers UAV mobility, energy, degree, and

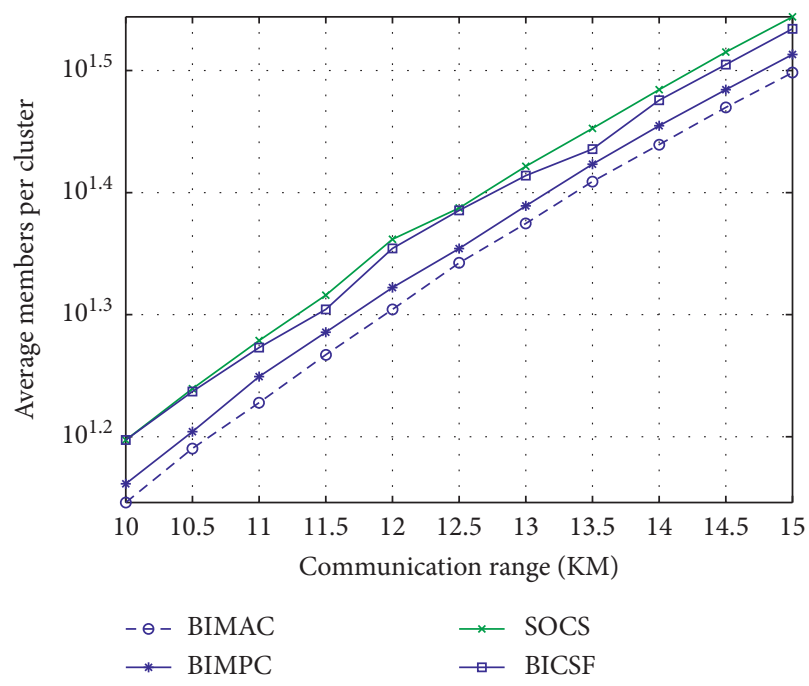

Figure 11: UAV degree versus maximum communication range.

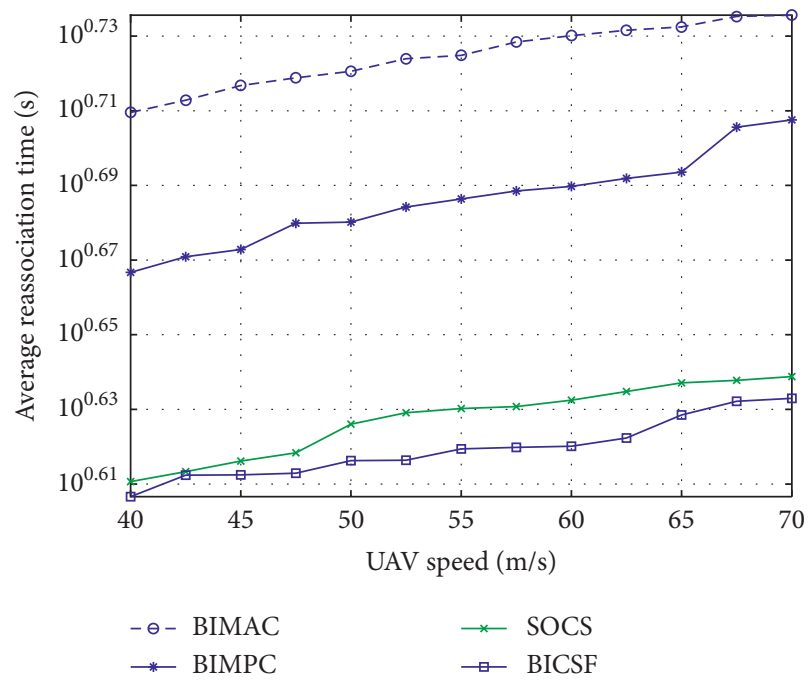

Figure 12: Average reassociation time versus UAV speed.

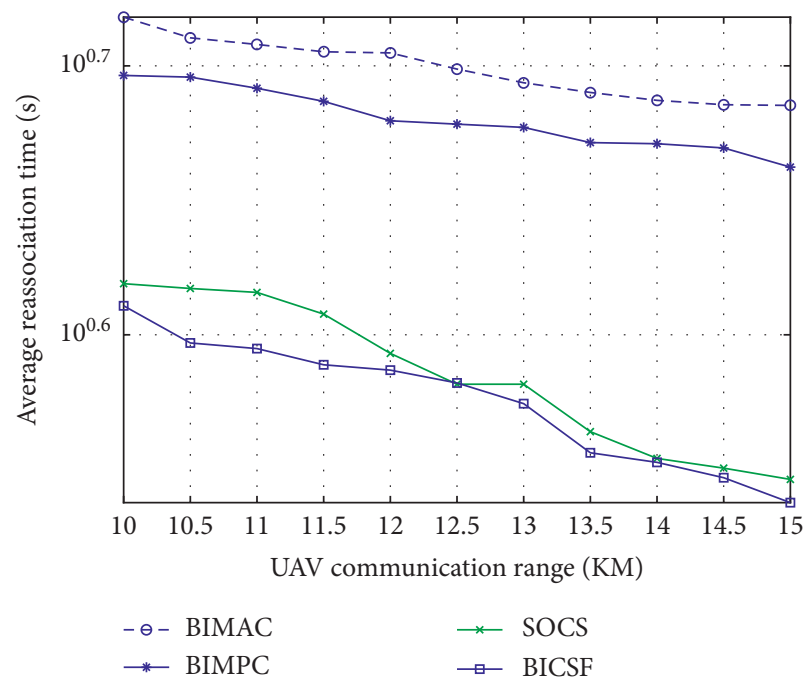

FIGURE 13: Average reassociation time versus UAV communication range. 


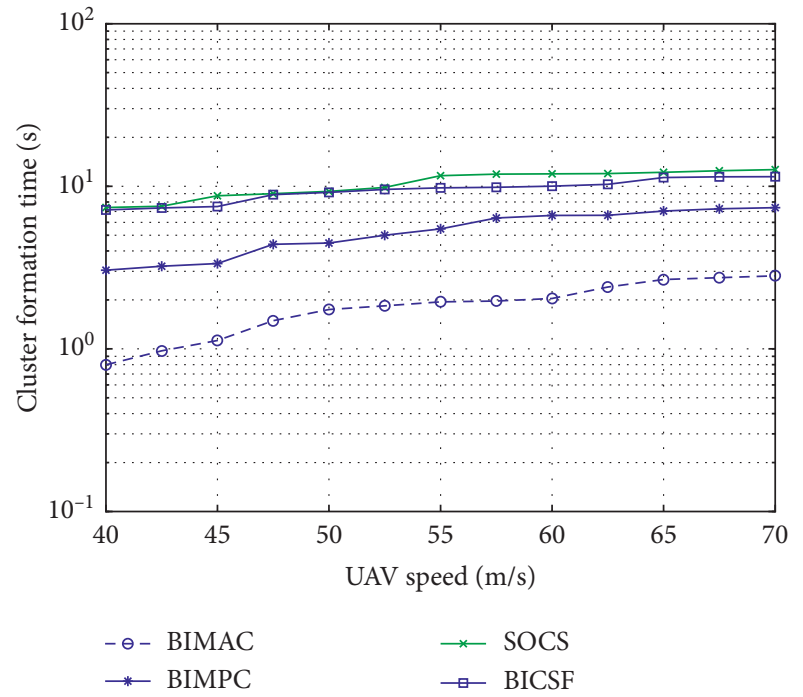

FIgURE 14: Cluster formation time versus UAV speed.

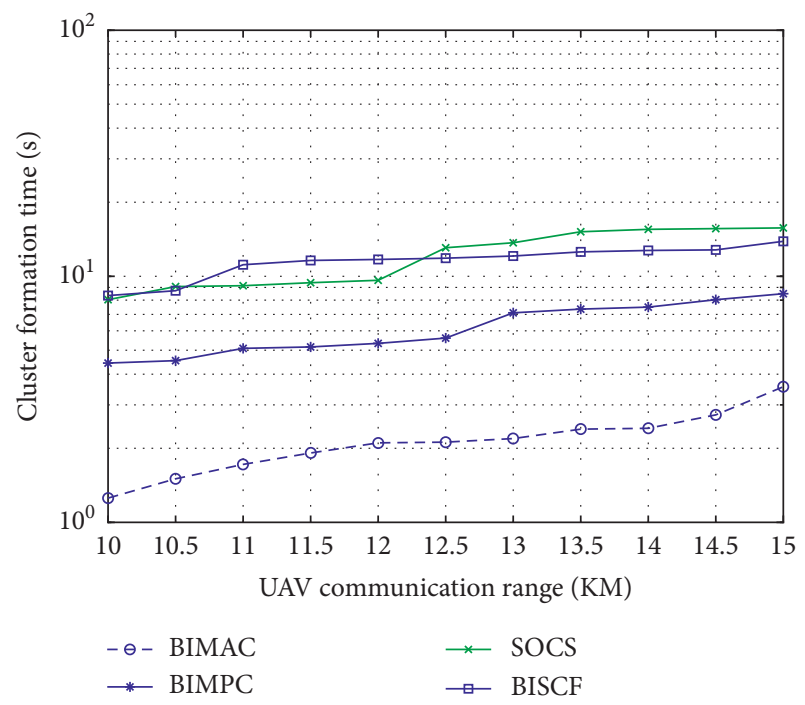

Figure 15: Cluster formation time versus UAV communication range.

communication load. The total time taken to form a cluster is known as cluster formation time. The $\mathrm{CH}$ selection and the membership of UAVs in the clustering process of the proposed algorithm minimize the cost in terms of time for balance cluster formation. The simulation result in Figure 14 represents the UAV speed and the cluster formation time for the proposed BIMAC, BIMPC [40], SOCS [41], and BICSF [42] algorithms. The UAV speed considered is $55 \mathrm{~m} / \mathrm{s}$. With the increase in UAV speed towards the maximum limit, the proposed BIMAC algorithm has still minimum cluster time than other selected algorithms, i.e., [40-42]. The reason is the use of multiple parameters in BIMAC as mentioned prior in this section.

The simulation results in Figure 15 show the cluster building time and communication range for the BIMAC, BIMPC [40], SOCS [41], and BICSF [42] algorithms. The
UAV communication range is considered $10 \mathrm{~km}$. With the increase in UAV communication range towards the maximum limit, the proposed algorithm BIMAC shows less variation in cluster formation time, as compared to the other selected algorithms in [40-42].

\section{Conclusion and Future Directions}

With the advancement of sensor and flying nodes technology, the development of low-cost flying nodes becomes easy. The world is moving towards real applications of IoT network; the flying segment of IoT network is demanding area for various applications. In this paper, the recent developments in FASNET with a special focus on cluster-based swarm optimization for IoTs have been studied. The latest trends in the area have been analyzed, and the future directions are stated. A taxonomy has been presented on the basis of different swarm intelligence techniques for cluster formation. Futuristic clustering schemes have been analyzed. The major findings of each scheme have been stated. The authors focus on part of optimization problem for clustering in FASNET such as node degree or mobility or remaining energy and communication load. According to the author's knowledge, none of the existing schemes had focused on all the parameters mentioned above. It is our recommendation that at least mobility, energy, and degree of nodes may be considered during the $\mathrm{CH}$ selection process.

In this paper, a bioinspired mobility-aware clustering optimization in flying ad hoc sensor network for internet of things BIMAC-FASNET has been proposed. The clusters in FASNET are formed using the honey bee optimization algorithm. The $\mathrm{CHs}$ are selected on the basis of UAVs relative mobility, degree, and remaining energy. The member UAVs join the nearest $\mathrm{CH}$ in the vicinity. In case of tie, a UAV can join a $\mathrm{CH}$ randomly. The simulation results show that our proposed scheme outperforms existing schemes in terms of link connection lifetime, reaffiliation rate, communication load, number of UAVs per cluster, $\mathrm{CH}$ lifetime, and cluster formation time.

The research will help academia to explore new areas of research. More researchers may be attracted to unfold and solve the issues in smart agriculture, intrusion detection, smart cities, smart transportations, and smart buildings using low-cost flying sensors. The society will benefit by using advance technology and work remotely with ease.

\section{Data Availability}

The data used to support the findings of this research are included within the article.

\section{Disclosure}

The authors received no financial support for the research and publication of this article.

\section{Conflicts of Interest}

The authors declare that there are no conflicts of interest associated with this publication. 


\section{References}

[1] W. Qi, Q. Song, X. Kong, and L. Guo, “A traffic-differentiated routing algorithm in flying ad hoc sensor networks with SDN cluster controllers," Journal of the Franklin Institute, vol. 356, 2017.

[2] S.-J. Yoo, J.-H. Park, S.-H. Kim, and A. Shrestha, "Flying path optimization in uav-assisted IoT sensor networks," ICT Express, vol. 2, no. 3, pp. 140-144, 2016.

[3] V. Mochalov and A. Pschenichnikov, "Functional scheme of the flying sensor networks architecture design," in Proceedings of the 2016 18th International Conference on Advanced Communication Technology (ICACT), pp. 659-663, Pyeongchang, South Korea, January 2016.

[4] M. Y. Arafat and S. Moh, "A survey on cluster-based routing protocols for unmanned aerial vehicle networks," IEEE Access, vol. 7, pp. 498-516, 2019.

[5] A. Mehmood, J. L. Mauri, M. Noman, and H. Song, "Improvement of the wireless sensor network lifetime using leach with vice-cluster head," Ad Hoc \& Sensor Wireless Networks, vol. 28, pp. 1-17, 2015.

[6] W. Zafar and B. Muhammad Khan, "Flying ad-hoc networks: technological and social implications," IEEE Technology and Society Magazine, vol. 35, no. 2, pp. 67-74, 2016.

[7] E. Kuiper and S. Nadjm-Tehrani, "Mobility models for UAV group reconnaissance applications," in International Conference on Wireless and Mobile Communications (ICWMC'06), Bucharest, Romania, July 2006.

[8] C. C. Miguel Itallo, B. Azevedo, E. M. Toda, T. C. Carvalho, and J. Jailton, "Wireless communications challenges to flying ad hoc networks (FANET)," Mobile Computing, IntechOpen, London, UK, 2019.

[9] Y.-H. Ho, Y.-R. Chen, and L.-J. Chen, "Krypto: assisting search and rescue operations using wi-fi signal with UAV," in Proceedings of the First Workshop on Micro Aerial Vehicle Networks, Systems, and Applications for Civilian Use, pp. 3-8, Florence, Italy, May 2015.

[10] M. Erdelj and E. Natalizio, "UAV-assisted disaster management: applications and open issues," in Proceedings of the International Conference on Computing, Networking and Communications (ICNC), Kauai, HI, USA, February 2016.

[11] T. Samad, J. S. Bay, and D. Godbole, "Network-centric systems for military operations in urban terrain: the role of UAVs," Proceedings of the IEEE, vol. 95, no. 1, pp. 92-107, 2007.

[12] R. Reshma, T. Ramesh, and P. Sathishkumar, "Security situational aware intelligent road traffic monitoring using UAVs," in Proceedings of the International Conference on VLSI Systems, Architectures, Technology and Applications (VLSI-SATA), pp. 1-6, Bangalore, India, January 2016.

[13] O. K. Sahingoz, "Networking models in flying ad-hoc networks (FANETs): concepts and challenges," Journal of Intelligent \& Robotic Systems, vol. 74, no. 1-2, pp. 513-527, 2014.

[14] L. Gupta, R. Jain, and G. Vaszkun, "Survey of important issues in UAV communication networks," IEEE Communications Surveys \& Tutorials, vol. 18, no. 2, pp. 1123-1152, 2016.

[15] O. S. Oubbati, A. Lakas, F. Zhou, M. Güneş, and M. B. Yagoubi, "A survey on position-based routing protocols for flying ad hoc networks (FANETs)," Vehicular Communications, vol. 10, pp. 29-56, 2017.

[16] J. Jiang and G. Han, "Routing protocols for unmanned aerial vehicles," IEEE Communications Magazine, vol. 56, no. 1, pp. 58-63, 2018.

[17] A. Mukherjee, V. Keshary, K. Pandya, N. Dey, and S. C. Satapathy, "Flying ad hoc networks: a comprehensive survey," in Information and Decision Sciences, pp. 569-580, Springer, Berlin, Germany, 2018.

[18] F. Yang, P. Wang, Y. Zhang, L. Zheng, and J. Lu, "Survey of swarm intelligence optimization algorithms," in Proceedings of the IEEE International Conference on Unmanned Systems (ICUS), pp. 544-549, Beijing, China, 2017.

[19] Y. Sun, "Physarum-inspired network optimization: a review," 2017, https://arxiv.org/pdf/1712.02910.pdf.

[20] K. N. Krishnanand and D. Ghose, "Glowworm swarm optimisation: a new method for optimising multi-modal functions," International Journal of Computational Intelligence Studies, vol. 1, no. 1, pp. 93-119, 2009.

[21] S. Mirjalili, S. M. Mirjalili, and A. Lewis, "Grey wolf optimizer," Advances in Engineering Software, vol. 69, pp. 46-61, 2014.

[22] A. Colorni, M. Dorigo, and V. Maniezzo, "Distributed optimization by ant colonies," in Proceedings of the First European Conference on Artificial Life, pp. 134-142, Cambridge, MA, USA, 1992.

[23] H. A. Abbass, "MBO: marriage in honey bees optimization-a haplometrosis polygynous swarming approach," in Proceedings of the 2001 Congress on Evolutionary Computation, Seoul, South Korea, May 2001.

[24] D. Karaboga, "An idea based on honey bee swarm for numerical optimization,"Technical Report, Kayseri, Turkey.

[25] F. L. Howard, "The life history ofphysarum polycephalum," American Journal of Botany, vol. 18, no. 2, pp. 116-133, 1931.

[26] Z. Li and X. Huang, "Glowworm swarm optimization and its application to blind signal separation," Mathematical Problems in Engineering, vol. 2016, Article ID 5481602, 8 pages, 2016.

[27] F. Bai and A. Helmy, "A survey of mobility models in wireless adhoc networks," 2004.

[28] D. B. Johnson and D. A. Maltz, "Dynamic source routing in ad hoc wireless networks," in Mobile Computing, pp. 153-181, Springer, Berlin, Germany, 1996.

[29] R. R. Roy, "Mobility model characteristics," in Handbook of Mobile Ad Hoc Networks for Mobility Models, pp. 23-32, Springer, Berlin, Germany, 2011.

[30] K. Pearson, "The problem of the random walk," Nature, vol. 72, no. 1867, p. 342, 1905.

[31] E. M. Royer, P. M. Melliar-Smith, and L. E. Moser, "An analysis of the optimum node density for ad hoc mobile networks," in Proceedings of the IEEE International Conference on Communications, pp. 857-861, Helsinki, Finland, June 2001.

[32] A. Bujari, C. T. Calafate, J.-C. Cano, P. Manzoni, C. E. Palazzi, and D. Ronzani, "Flying ad-hoc network application scenarios and mobility models," International Journal of Distributed Sensor Networks, vol. 13, Article ID 1550147717738192, 2017.

[33] B. Liang and Z. J. Haas, "Predictive distance-based mobility management for PCS networks," in Proceedings of the IEEE INFOCOM'99. Conference on Computer Communications. Proceedings. Eighteenth Annual Joint Conference of the IEEE Computer and Communications Societies, pp. 1377-1384, New York, NY, USA, March 1999.

[34] T. Camp, J. Boleng, and V. Davies, "A survey of mobility models for ad hoc network research," Wireless Communications and Mobile Computing, vol. 2, no. 5, pp. 483-502, 2002.

[35] K. Kumari, B. Sah, and S. Maakar, "A survey: different mobility model for fanet," International Journal of Advanced Research in Computer Science and Software Engineering, vol. 5, 2015. 
[36] O. Bouachir, A. Abrassart, F. Garcia, and N. Larrieu, "A mobility model for UAV ad hoc network," in Proceedings of the International Conference on Unmanned Aircraft Systems (ICUAS), pp. 383-388, Orlando, FL, USA, May 2014.

[37] M. Sánchez and P. Manzoni, “Anejos: a java based simulator for ad hoc networks," Future Generation Computer Systems, vol. 17, no. 5, pp. 573-583, 2001.

[38] J. Sanchez-Garcia, J. Garcia-Campos, S. Toral, D. Reina, and F. Barrero, "A self organising aerial ad hoc network mobility model for disaster scenarios," in Proceedings of the International Conference on Developments of E-Systems Engineering (DeSE), pp. 35-40, Dubai, UAE, December 2015.

[39] M.-A. Messous, S.-M. Senouci, and H. Sedjelmaci, "Network connectivity and area coverage for UAV fleet mobility model with energy constraint," in Proceedings of theIEEE Wireless Communications and Networking Conference, pp. 1-6, Doha, Qatar, April 2016.

[40] Y. Yu, L. Ru, and K. Fang, "Bio-inspired mobility prediction clustering algorithm for ad hoc UAV networks," Engineering Letters, vol. 24, 2016.

[41] A. Khan, F. Aftab, and Z. Zhang, "Self-organization based clustering scheme for FANETs using glowworm swarm optimization," Physical Communication, vol. 36, Article ID 100769, 2019.

[42] A. Khan, F. Aftab, and Z. Zhang, "BICSF: bio-inspired clustering scheme for fanets," IEEE Access, vol. 7, pp. 31446-31456, 2019.

[43] Q. Yu, N. Zhang, W. Meng, and F. Adachi, "A novel stability weighted clustering algorithm for multi-hop packet radio virtual cellular network," in Proceedings of the IEEE Wireless Communication and Networking Conference, pp. 1-6, Sydney, Australia, April 2010.

[44] W. Zheng and J. Shu, "An improved artificial bee colonybased relocation algorithm in mobile sensor network," Information Engineering Letters, vol. 2, p. 17, 2012.

[45] M. Abd-El-Barr, "Ant colony heuristic algorithm for multilevel synthesis of multiple-valued logic functions," IAENG International Journal of Computer Science, vol. 37, pp. 1-7, 2010.

[46] K. Liu, J. Zhang, and T. Zhang, "The clustering algorithm of UAV networking in near-space," in Proceedings of the 8th International Symposium on Antennas, Propagation and EM Theory, pp. 1550-1553, Kunming, China, November 2008.

[47] N. Al-Aboody and H. Al-Raweshidy, "Grey wolf optimization-based energy-efficient routing protocol for heterogeneous wireless sensor networks," in Proceedings of the 4th International Symposium on Computational and Business Intelligence (ISCBI), pp. 101-107, Olten, Switzerland, September 2016.

[48] V. A. Maistrenko, L. V. Alexey, and V. A. Danil, "Experimental estimate of using the ant colony optimization algorithm to solve the routing problem in fanet," in Proceedings of the International Siberian Conference on Control and Communications (SIBCON), pp. 1-10, Moscow, Russia, June 2016.

[49] J. Yang, X. Wang, Z. Li et al., "Path planning of unmanned aerial vehicles for farmland information monitoring based on WSN," in Proceedings of the 12th World Congress on Intelligent Control and Automation (WCICA), pp. 2834-2838, Guilin, China, June 2016.

[50] N. E. H. Bahloul, S. Boudjit, M. Abdennebi, and D. E. Boubiche, "A flocking-based on demand routing protocol for unmanned aerial vehicles," Journal of Computer Science and Technology, vol. 33, pp. 263-276, 2018.
[51] J. Wang, Y.-Q. Cao, B. Li, S.-Y. Lee, and J.-U. Kim, “A glowworm swarm optimization based clustering algorithm with mobile sink support for wireless sensor networks," Journal of Internet Technology, vol. 16, pp. 825-832, 2015.

[52] A. V. Leonov, "Modeling of bio-inspired algorithms anthocnet and beeadhoc for flying ad hoc networks (fanets)," in Proceedings of the 13th International Scientific-Technical Conference on Actual Problems of Electronics Instrument Engineering (APEIE), pp. 90-99, Novosibirsk, Russia, October 2016.

[53] A. V. Leonov, "Application of bee colony algorithm for fanet routing," in Proceedings of the 17th International Conference of Young Specialists on Micro/Nanotechnologies and Electron Devices (EDM), pp. 124-132, Erlagol, Russia, July 2016.

[54] M. Ahmad, A. Hameed, F. Ullah, I. Wahid, S. U. Rehman, and H. A. Khattak, "A bio-inspired clustering in mobile adhoc networks for internet of things based on honey bee and genetic algorithm," Journal of Ambient Intelligence and $\mathrm{Hu}$ manized Computing, vol. 2018, pp. 1-15, 2018.

[55] M. Ahmad, A. A. Ikram, I. Wahid, M. Inam, N. Ayub, and S. Ali, "A bio-inspired clustering scheme in wireless sensor networks: beewsn," Procedia Computer Science, vol. 130, pp. 206-213, 2018.

[56] N. Khatoon, "Mobility aware energy efficient clustering for MANET: a bio-inspired approach with particle swarm optimization," Wireless Communications and Mobile Computing, vol. 2017, Article ID 1903190, 12 pages, 2017.

[57] Z. Han, A. L. Swindlehurst, and K. R. Liu, "Optimization of manet connectivity via smart deployment/movement of unmanned air vehicles," IEEE Transactions on Vehicular Technology, vol. 58, pp. 3533-3546, 2009.

[58] A. M. S. Abdelgader, L. Wu, and M. M. M. Nasr, "A simplified mobile ad hoc network structure for helicopter communication," International Journal of Aerospace Engineering, vol. 201615 pages, Article ID 2132941, 2016. 